Partially coherent electromagnetic beams propagating through double-wedge depolarizers

This content has been downloaded from IOPscience. Please scroll down to see the full text.

2014 J. Opt. 16035708

(http://iopscience.iop.org/2040-8986/16/3/035708)

View the table of contents for this issue, or go to the journal homepage for more

Download details:

IP Address: 147.96.14.16

This content was downloaded on 05/05/2014 at 18:19

Please note that terms and conditions apply. 


\title{
Partially coherent electromagnetic beams propagating through double-wedge depolarizers
}

\author{
J Carlos G de Sande ${ }^{1}$, Gemma Piquero ${ }^{2}$, Massimo Santarsiero ${ }^{3}$ and \\ Franco Gori ${ }^{3}$ \\ ${ }^{1}$ Departamento de Circuitos y Sistemas, Universidad Politécnica de Madrid, Campus Sur, E-28031 \\ Madrid, Spain \\ ${ }^{2}$ Departamento de Óptica, Universidad Complutense de Madrid, E-28040 Madrid, Spain \\ ${ }^{3}$ Dipartimento di Ingegneria, Università Roma Tre, and CNISM, Via V. Volterra 62, I-00146 Rome, Italy \\ E-mail: jcgsande@ics.upm.es
}

Received 26 November 2013, revised 11 January 2014

Accepted for publication 14 January 2014

Published 3 March 2014

\begin{abstract}
The irradiance and polarization characteristics of quasi-monochromatic partially coherent electromagnetic beams are analyzed when they propagate after passing through a deterministic linear optical element, i.e., an optical element that can be represented by a Jones matrix. A class of such optical elements, which includes double-wedge depolarizers and polarization gratings, is defined and studied in detail. Analytical expressions are obtained for the case of double-wedge depolarizers and examples are given for an incident Gaussian Schell-model beam. For such an input beam, the effects on the irradiance and degree of polarization of the field propagating beyond the optical element are investigated in detail. A rich variety of behaviors is obtained by varying the beam size, coherence width and polarization state of the input field. The results not only provide a mathematical extension of well-known results to the domain of partial coherence, but they also exemplify mixing between coherence and polarization, which is, of course, not possible if, for example, fully spatially coherent fields are analyzed.
\end{abstract}

Keywords: coherence, polarization, partially coherent electromagnetic sources

PACS numbers: 42.25.Ja, 42.25.Kb

(Some figures may appear in colour only in the online journal)

\section{Introduction}

There is presently an increasing interest in optical elements that reduce the degree of polarization of monochromatic light beams, in order to use them in those applications where a low degree of polarization is required for an optimum performance of an optical system [1-10]. Among these devices we mention the double-wedge depolarizer (DWD), which consists of a pair of uniaxial crystal wedges having their optic axes mutually out of line $[1,9]$. The depolarization effect of DWDs is based on scrambling the states of polarization in the space domain, so they are actually pseudodepolarizers. DWDs have been studied by considering incident monochromatic plane waves, in both totally polarized and unpolarized cases, and interesting results have been obtained [9]. To our knowledge, other types of incident beams, such as partially coherent beams, have not been considered yet.

A significant class of partially polarized fields is that of the so-called purely polarized fields, whose spatial correlation properties and polarization states can be decoupled [11-13]. Fields belonging to this class present a uniform polarization across the transverse plane and their polarization characteristics are invariant under propagation through any polarization- 
insensitive optical system and, as a particular case, during free propagation [13].

The aim of this work is to investigate the effect of a DWD on the propagation of a purely polarized partially coherent beam. We limit ourselves to the case of quasi-monochromatic radiation, but the extension to the polychromatic case is straightforward. The results we are going to present can also be applied to a wider class of deterministic optical elements, namely, those that split an incoming field into two or more components, on introducing a different phase for each component that depends linearly on the transverse position. Besides DWDs [9], this class of optical elements also includes polarization gratings $[14,15]$ and any other optical element that produces a transversely periodic electromagnetic field [16]. Nonetheless, the particular case of DWDs is fully developed in our work.

Numerical examples are given for purely polarized input beams of the Gaussian Schell-model (GSM) type, for which both the irradiance profile and the coherence degree have a Gaussian form [14, 17-22]. For the two extreme cases of totally polarized and completely unpolarized input fields, the irradiance and polarization characteristics are analyzed in detail. The irradiance and the degree of polarization just at the output of the DWD and after free propagation are studied as functions of the parameters of both the input beam and the optical device. It is shown that, by varying the input beam characteristics, a rich variety of behaviors can be obtained not only across the transverse beam section but also upon propagation. Extension of the numerical examples to the case of polarization gratings is straightforward.

The paper is structured as follows. The mathematical tools used throughout the paper are introduced in section 2: in particular, the beam coherence-polarization (BCP) matrix, for treating partially coherent electromagnetic beams, and the Jones formalism, for studying the effects of a DWD on an incident field. In section 3 , the BCP of the field propagating after the DWD is derived, while in section 4 examples are given concerning the irradiance distribution and the degree of polarization of purely polarized GSM input beams after passing through a DWD. The two limiting cases of totally polarized and completely unpolarized input beams are considered in detail, for different choices of the beam parameters. The main conclusions of this work are summarized in section 5. Moreover, in appendix A all the symbols and notations used throughout the text are summarized, while the analytical derivations of some of the expressions used in the paper are reported in four additional appendices.

\section{Preliminaries}

\subsection{The beam coherence-polarization matrix}

Within the paraxial approximation, the coherence and polarization characteristics of a quasi-monochromatic light beam, propagating along the $z$ direction of a suitable reference frame, can be described by means of its BCP matrix [23-25], defined as

$$
\hat{J}\left(\mathbf{r}_{1}, \mathbf{r}_{2}, z\right)=\left(\begin{array}{ll}
J_{x x}\left(\mathbf{r}_{1}, \mathbf{r}_{2}, z\right) & J_{x y}\left(\mathbf{r}_{1}, \mathbf{r}_{2}, z\right) \\
J_{y x}\left(\mathbf{r}_{1}, \mathbf{r}_{2}, z\right) & J_{y y}\left(\mathbf{r}_{1}, \mathbf{r}_{2}, z\right)
\end{array}\right),
$$

where

$$
J_{j k}\left(\mathbf{r}_{1}, \mathbf{r}_{2}, z\right)=\left\langle E_{j}\left(\mathbf{r}_{1}, z ; t\right) E_{k}^{*}\left(\mathbf{r}_{2}, z ; t\right)\right\rangle .
$$

Here, $E_{j}(\mathbf{r}, z ; t)$, with $j, k=x, y$, are the Cartesian components of the time-dependent electric field and $\mathbf{r}$ is the position vector in a plane perpendicular to the $z$ direction. The asterisk denotes conjugation and the angle brackets represent temporal average. In the case of polychromatic fields, the cross-spectral density tensor defined in the spectral domain $[21,26]$ should be used, but the two definitions are equivalent if quasi-monochromatic sources are considered.

When evaluated at coincident points, $\mathbf{r}_{1}=\mathbf{r}_{2}=\mathbf{r}$, the BCP matrix reduces to the polarization matrix, i.e.,

$$
\hat{P}(\mathbf{r}, z)=\hat{J}(\mathbf{r}, \mathbf{r}, z),
$$

containing all information about the irradiance and polarization state of the beam at the point $(\mathbf{r}, z)$. In particular, the total irradiance is defined as the trace of $\hat{P}$, while the local degree of polarization (DoP) is evaluated as [21]

$$
p(\mathbf{r})=\sqrt{1-\frac{4 \operatorname{Det}\{\hat{P}(\mathbf{r})\}}{\operatorname{Tr}^{2}\{\hat{P}(\mathbf{r})\}}} .
$$

Since the matrix $\hat{P}$ is Hermitian and semipositive definite, the DoP only takes values in the interval $[0,1]$, with $p=1$ corresponding to a perfectly polarized field and $p=0$ to a completely unpolarized one.

The polarization properties of the light fields can be also described through the Stokes vector, which conveys the same information content as the polarization matrix, and whose elements are related to the elements of the latter by the relation

$$
\mathbf{S}(\mathbf{r})=\left(\begin{array}{l}
S_{0}(\mathbf{r}) \\
S_{1}(\mathbf{r}) \\
S_{2}(\mathbf{r}) \\
S_{3}(\mathbf{r})
\end{array}\right)=\left(\begin{array}{c}
P_{x x}(\mathbf{r})+P_{y y}(\mathbf{r}) \\
P_{x x}(\mathbf{r})-P_{y y}(\mathbf{r}) \\
2 \operatorname{Re}\left\{P_{y x}(\mathbf{r})\right\} \\
2 \operatorname{Im}\left\{P_{y x}(\mathbf{r})\right\}
\end{array}\right) .
$$

$S_{0}(\mathbf{r})$ gives the irradiance of the field while the three remaining parameters account for its local polarization state. The Stokes parameters are measurable quantities and can be determined from the irradiances at the output of a quarter wave phase plate and a linear polarizer at different angles [27].

When a partially coherent electromagnetic field passes thorough a deterministic linear optical element, characterized by a complex $2 \times 2$ Jones matrix $\hat{T}(\mathbf{r})$, its BCP matrix is transformed according to the following rule [24]:

$$
\hat{J}\left(\mathbf{r}_{1}, \mathbf{r}_{2}, 0\right)=\hat{T}\left(\mathbf{r}_{1}\right) \hat{J}^{\text {in }}\left(\mathbf{r}_{1}, \mathbf{r}_{2}, 0\right) \hat{T}^{\dagger}\left(\mathbf{r}_{2}\right),
$$

where $\hat{J}^{\text {in }}$ is the BCP matrix across the input plane of the element and the dagger denotes Hermitian conjugation. It has been assumed that the optical element has negligible thickness and its exit plane coincides with the plane $z=0$. Equation (6) can be written for each of the matrix elements of the $\mathrm{BCP}$ matrix as

$$
J_{j k}\left(\mathbf{r}_{1}, \mathbf{r}_{2}, 0\right)=\sum_{l, m=x}^{y} T_{j l}\left(\mathbf{r}_{1}\right) T_{k m}^{*}\left(\mathbf{r}_{2}\right) J_{l m}^{\text {in }}\left(\mathbf{r}_{1}, \mathbf{r}_{2}, 0\right) .
$$




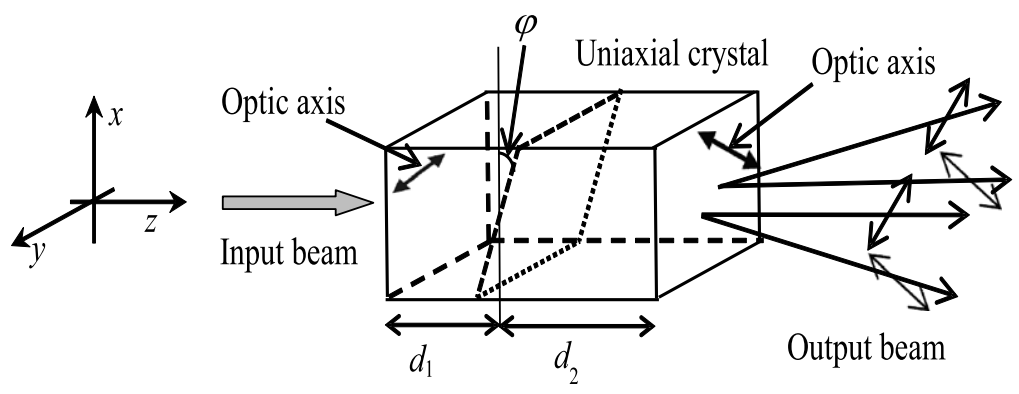

Figure 1. Scheme of the double-wedge depolarizer.

It must be recalled that, in general, a deterministic linear optical element not only changes the polarization state of the polarized part of the input beam, but also changes the DoP of the input beam [28]. Specifically, the DoP at the exit plane is set to unity for polarizers (singular Jones matrices), while it remains unchanged when the Jones matrix of the optical element is proportional to a unitary matrix. Otherwise, the DoP can be increased, decreased or unchanged by optical elements represented by nonsingular Jones matrices that are not proportional to a unitary matrix.

In this work, particular attention will be focused on input beams belonging to the class of the so-called purely polarized fields [11-13], i.e., those fields represented by BCP matrices of the form

$$
\hat{J}^{\text {in }}\left(\mathbf{r}_{1}, \mathbf{r}_{2}, 0\right)=J_{\mathrm{sc}}\left(\mathbf{r}_{1}, \mathbf{r}_{2}, 0\right) \hat{P}^{\text {in }}
$$

where $J_{\mathrm{sc}}\left(\mathbf{r}_{1}, \mathbf{r}_{2}, 0\right)$ is the mutual intensity of a scalar source at the plane $z=0$ and $\hat{P}^{\text {in }}$ is a normalized polarization matrix, giving account of the polarization state. Fields of this class are uniformly polarized and their polarization characteristics are invariant under propagation through polarization-insensitive optical systems, such as propagation in free space [13]. When a beam of this class impinges onto a deterministic linear optical element, the elements of the BCP matrix across the output plane become

$J_{j k}\left(\mathbf{r}_{1}, \mathbf{r}_{2}, 0\right)=J_{\mathrm{sc}}\left(\mathbf{r}_{1}, \mathbf{r}_{2}, 0\right) \sum_{l, m=x}^{y} T_{j l}\left(\mathbf{r}_{1}\right) T_{k m}^{*}\left(\mathbf{r}_{2}\right) P_{l m}^{\mathrm{in}}$.

Since, in general, the Jones matrix $\hat{T}$ is a function of the transverse coordinate $\mathbf{r}$, the output field is no longer purely polarized, implying that the polarization properties of the field after the optical element may vary under free-space propagation. Of course, this does not happen in the case of optical elements that act uniformly across the transverse section of a purely polarized beam.

\subsection{Double-wedge depolarizer}

A DWD consists of two birefringent wedges with the same wedge angle $\varphi$,joined in such a way as to form a parallelepiped (see figure 1). The first wedge has its optic axis along the $y$ direction. The optic axis of the second wedge is parallel to the bisector of the $x$ and $y$ axes. When a plane wave impinges perpendicularly to the input face of a DWD, the ordinary and extraordinary components of the field propagate through the first wedge and both of them split into two new ordinary and extraordinary components in the second one [29, 30]. Two of these components propagate, after the DWD, following the same direction as the incident plane wave but with different phases and orthogonal polarizations. The two other components, also having mutually orthogonal polarization states, diverge, forming a very small angle (if compared to $\varphi$ ) with respect to the $z$ axis (see figure 1). We will neglect the losses due to reflections at the interfaces and the small differences between the transmission coefficients for ordinary and extraordinary waves or between the parallel and perpendicular components at the exit face.

Under the above hypothesis, the behavior of the DWD can be described by the following Jones matrix [9]:

$\hat{T}^{\mathrm{D}}(x)=\frac{1}{2}\left(\begin{array}{ll}1+\mathrm{e}^{-\mathrm{i} \delta_{2}(x)} & \mathrm{e}^{-\mathrm{i} \delta_{1}(x)}\left[1-\mathrm{e}^{-\mathrm{i} \delta_{2}(x)}\right] \\ 1-\mathrm{e}^{-\mathrm{i} \delta_{2}(x)} & \mathrm{e}^{-\mathrm{i} \delta_{1}(x)}\left[1+\mathrm{e}^{-\mathrm{i} \delta_{2}(x)}\right]\end{array}\right)$.

In this equation, $\delta_{1}$ and $\delta_{2}$ are the phase differences between the $x$ and $y$ components of the field after propagating through the first and second wedge of the DWD, respectively. It can be noted that $\hat{T}^{\mathrm{D}}(x)$ is a unitary matrix, therefore the DWD only changes the polarization state in a different way at each point of the beam transverse section, but it does not modify its irradiance and DoP.

For small wedge angles $(\varphi \ll 1)$, the above phase differences can be approximated by [9]

$$
\begin{aligned}
& \delta_{1}(x) \simeq \frac{2 \pi}{\lambda}|\Delta n|\left(d_{1}+x \tan \varphi\right)=\delta_{10}+\gamma x, \\
& \delta_{2}(x) \simeq \frac{2 \pi}{\lambda}|\Delta n|\left(d_{2}-x \tan \varphi\right)=\delta_{20}-\gamma x,
\end{aligned}
$$

where $d_{1}$ and $d_{2}$ are the mean thicknesses of the first and second wedge, respectively, $\gamma=(2 \pi|\Delta n| \tan \varphi) / \lambda$, and $\Delta n=n_{\mathrm{o}}-$ $n_{\mathrm{e}}$, with $n_{\mathrm{o}}$ and $n_{\mathrm{e}}$ being the ordinary and extraordinary refractive indexes, respectively. It can be noticed that both phases are linear functions of the $x$ coordinate. The explicit dependence of the refractive indexes on the wavelength has been omitted. As is evident from equations (10)-(12), the geometry of the DWD yields a Jones matrix that depends only on the $x$ coordinate, so that the same polarization state 
is expected at all points along lines with constant $x$ across the exit plane.

It can be shown that a DWD converts a uniformly totally polarized field into a non-uniformly totally polarized beam [9]. The local degree of polarization is unity everywhere, across the transverse section, but the state of polarization changes periodically along the $x$ direction with a spatial periodicity given by

$$
L_{x}=\frac{\lambda}{|\Delta n(\lambda)| \tan \varphi}=\frac{2 \pi}{\gamma} .
$$

Several experimental methods have been proposed to obtain periodic variation of the state of polarization across the transverse plane $[3,4,14,31]$. The use of a DWD is a simple way to obtain such a periodic variation without modifying the irradiance profile of the beam. If the DWD is illuminated with a totally linearly polarized plane wave (impinging perpendicularly to the input face of the DWD), a manifestation of the Talbot effect is observed, with selfreplication of the polarization states at integer multiples of the Talbot distance $z_{\mathrm{T}}=2 L_{x}^{2} / \lambda[16]$.

It is interesting to analyze the behavior of a DWD within the Stokes formalism. Starting from the expression of the Jones matrix in equation (10), it is possible to evaluate the pertinent Müller matrix [32, 33], which turns out to be

$$
\begin{aligned}
& \hat{M}^{\mathrm{D}}(x)=\hat{T}^{\mathrm{D}}(x) \otimes \hat{T}^{\mathrm{D} *}(x)= \\
& \left(\begin{array}{cccc}
1 & 0 & 0 & 0 \\
0 & \cos \delta_{2}(x) & \sin \delta_{1}(x) \sin \delta_{2}(x) & -\cos \delta_{1}(x) \sin \delta_{2}(x) \\
0 & 0 & \cos \delta_{1}(x) & \sin \delta_{1}(x) \\
0 & \sin \delta_{2}(x) & -\sin \delta_{1}(x) \cos \delta_{2}(x) & \cos \delta_{1}(x) \cos \delta_{2}(x)
\end{array}\right),
\end{aligned}
$$

where $\otimes$ represents the direct or Kronecker product. It is evident that, on averaging the Müller matrix elements over sufficiently large distances along the $x$ direction, all the terms vanish except $M_{11}^{\mathrm{D}}$. Thus, in such conditions the behavior of the DWD mimics that of an ideal depolarizer [34].

Finally, let us consider the effects of a DWD on incident partially coherent electromagnetic light. In such a case, the relationship between the input and the output $\mathrm{BCP}$ matrices of the field is described by equation (7). Taking into account the explicit form of the Jones matrix in equation (10), the products $T_{j l}\left(\mathbf{r}_{1}\right) T_{k m}^{*}\left(\mathbf{r}_{2}\right)$, appearing in equation (7), turn out to be linear combinations of the phase terms introduced by the DWD, and such phases are, at least approximately, linear functions of the $x$ coordinate (see equations (11) and (12)). As will be seen in the next section, this feature makes simpler the evaluation of the BCP matrix of the field propagated from the DWD, once the propagation of the unperturbed input field (i.e., the field we would have obtained if the optical element were not present) is known. The explicit expressions for these products are given in appendix B.

\section{Free-space propagation after a deterministic linear optical element}

Knowledge of the BCP matrix across the exit plane of the optical element enables the evaluation of the $\mathrm{BCP}$ matrix across any transverse plane beyond it, such that the polarization properties of the propagated field can be determined. In particular, the following expression can be used for free propagation in paraxial conditions $[17,21]$ :

$$
\begin{aligned}
J_{j k}\left(\mathbf{r}_{1}, \mathbf{r}_{2}, z\right)= & \iint K_{z}^{*}\left(\mathbf{r}_{1}, \boldsymbol{\rho}_{1}\right) J_{j k}\left(\boldsymbol{\rho}_{1}, \boldsymbol{\rho}_{2}, 0\right) \\
& \times K_{z}\left(\mathbf{r}_{2}, \boldsymbol{\rho}_{2}\right) \mathrm{d}^{2} \rho_{1} \mathrm{~d}^{2} \rho_{2}
\end{aligned}
$$

where $\rho$ is the position vector across the $z=0$ plane and

$$
K_{z}(\mathbf{r}, \boldsymbol{\rho})=\frac{-\mathrm{i}}{\lambda z} \exp \left[\frac{\mathrm{i} 2 \pi z}{\lambda}+\frac{\mathrm{i} \pi}{\lambda z}(\mathbf{r}-\boldsymbol{\rho})^{2}\right]
$$

is the direct propagator in paraxial conditions.

On substituting from equation (16) into equation (15) and using equation (7), the BCP matrix elements of the propagated field can be expressed as

$$
\begin{aligned}
J_{j k}\left(\mathbf{r}_{1}, \mathbf{r}_{2}, z\right)= & \sum_{l, m=x}^{y} \frac{1}{\lambda^{2} z^{2}} \exp \left[\frac{\mathrm{i} \zeta}{2}\left(r_{2}^{2}-r_{1}^{2}\right)\right] \\
& \times \iint T_{j l}\left(\rho_{1}\right) T_{k m}^{*}\left(\boldsymbol{\rho}_{2}\right) J_{l m}^{\text {in }}\left(\rho_{1}, \boldsymbol{\rho}_{2}, 0\right) \\
& \times \exp \left[\frac { \mathrm { i } \zeta } { 2 } \left(\rho_{2}^{2}-\rho_{1}^{2}-2 \mathbf{r}_{2} \cdot \boldsymbol{\rho}_{2}\right.\right. \\
& \left.\left.+2 \mathbf{r}_{1} \cdot \rho_{1}\right)\right] \mathrm{d}^{2} \rho_{1} \mathrm{~d}^{2} \rho_{2},
\end{aligned}
$$

where $\zeta=2 \pi /(\lambda z)$ and $\cdot$ represents the scalar product

On the other hand, the free-space propagation of the unperturbed input field can be evaluated as

$$
J_{j k}^{\text {in }}\left(\mathbf{r}_{1}, \mathbf{r}_{2}, z\right)=\frac{\exp (\mathrm{i} \zeta \mathbf{s} \cdot \mathbf{t})}{\lambda^{2} z^{2}} H_{j k}(\mathbf{s}, \mathbf{t})
$$

where the auxiliary function $H_{j k}(\mathbf{s}, \mathbf{t})$ is introduced as

$$
\begin{aligned}
H_{j k}(\mathbf{s}, \mathbf{t})= & \iint J_{j k}^{\text {in }}\left(\boldsymbol{\rho}_{1}, \boldsymbol{\rho}_{2}, 0\right) \exp \left[\frac{\mathrm{i} \zeta}{2}\left(\rho_{2}^{2}-\rho_{1}^{2}\right)\right] \\
& \times \exp \left[-\mathrm{i} \zeta\left(\mathbf{r}_{2} \cdot \boldsymbol{\rho}_{2}-\mathbf{r}_{1} \cdot \boldsymbol{\rho}_{1}\right)\right] \mathrm{d}^{2} \rho_{1} \mathrm{~d}^{2} \rho_{2},
\end{aligned}
$$

with $\mathbf{s}=\left(\mathbf{r}_{1}+\mathbf{r}_{2}\right) / 2$ and $\mathbf{t}=\mathbf{r}_{2}-\mathbf{r}_{1}$. The explicit dependence of $H_{j k}$ on $z$ has been omitted for brevity.

In the following, particular attention will be paid to deterministic linear optical systems that can be represented by Jones matrices whose elements are linear combinations of phase terms, with phases that linearly depend on the position across the optical element plane. Such elements can be written in the form

$$
T_{j k}(\mathbf{r})=\sum_{q} a_{j k}^{q} \exp \left(\mathrm{i}_{j k}^{q} \cdot \mathbf{r}\right)
$$

where $a_{j k}^{q}$ are complex coefficients and the components of the vectors $\boldsymbol{\gamma}_{j k}^{q}$ give the proportionality along the $x$ and $y$ coordinates for each term. Jones matrices representing a DWD, equation (10), or a polarization grating [14, 15] belong to this class. For such class of optical elements it is found, in 
particular, that

$$
\begin{aligned}
T_{j l}\left(\boldsymbol{\rho}_{1}\right) T_{k m}^{*}\left(\boldsymbol{\rho}_{2}\right)= & \sum_{q, q^{\prime}} a_{j l}^{q}\left(a_{k m}^{q^{\prime}}\right)^{*} \\
& \times \exp \left[\mathrm{i} \boldsymbol{\gamma}_{j l}^{q} \cdot \boldsymbol{\rho}_{1}-\mathrm{i} \boldsymbol{\gamma}_{k m}^{q^{\prime}} \cdot \boldsymbol{\rho}_{2}\right] .
\end{aligned}
$$

Therefore, from equation (17), the BCP elements corresponding to the propagated field behind such an optical element can be expressed as

$$
\begin{aligned}
J_{j k}\left(\mathbf{r}_{1}, \mathbf{r}_{2}, z\right)= & \frac{1}{\lambda^{2} z^{2}} \exp \left[\frac{\mathrm{i} \zeta}{2}\left(r_{2}^{2}-r_{1}^{2}\right)\right] \\
& \times \sum_{l, m=x}^{y} j_{j k}^{l m}\left(\mathbf{r}_{1}, \mathbf{r}_{2}, z\right),
\end{aligned}
$$

where the functions

$$
\begin{aligned}
& j_{j k}^{l m}\left(\mathbf{r}_{1}, \mathbf{r}_{2}, z\right)=\sum_{q, q^{\prime}} a_{j l}^{q}\left(a_{j l}^{q^{\prime}}\right)^{*} \\
& \quad \times H_{l m}\left(\mathbf{s}-\frac{\boldsymbol{\gamma}_{j l}^{q}}{\zeta}+\frac{\boldsymbol{\gamma}_{k m}^{q^{\prime}}}{\zeta}, \mathbf{t}+\frac{\boldsymbol{\gamma}_{j l}^{q}}{2 \zeta}+\frac{\boldsymbol{\gamma}_{k m}^{q^{\prime}}}{2 \zeta}\right)
\end{aligned}
$$

are different linear combinations of the auxiliary functions $H_{l m}(\mathbf{s}, \mathbf{t})$ and different tilted versions of them. The particular set of linear combinations and tilts depends on the specific optical element considered. In appendix C, the particular case of a DWD is developed.

\section{Gaussian Schell-model beams through double-wedge depolarizers}

The process discussed in the previous section will be illustrated here for the case of a purely polarized GSM input beam [11-13]. This means that the BCP matrix of the input beam takes the form shown in equation (8), with the scalar mutual intensity given by $[17,35]$

$$
J_{\mathrm{sc}}\left(\mathbf{r}_{1}, \mathbf{r}_{2}, 0\right)=I_{0} \exp \left[-\frac{r_{1}^{2}+r_{2}^{2}}{4 \sigma^{2}}-\frac{\left(\mathbf{r}_{1}-\mathbf{r}_{2}\right)^{2}}{2 \mu^{2}}\right],
$$

where $I_{0}$ is a positive quantity having dimensions of an irradiance, $\sigma^{2}$ is the variance of the irradiance profile, and $\mu$ expresses the width of the degree of coherence of the beam across the input plane of the DWD.

For the case of a GSM beam, the explicit form of the auxiliary function $H(\mathbf{s}, \mathbf{t})$ given in equation (19) is [14]

$H(\mathbf{s}, \mathbf{t})=I_{0}\left(\frac{\lambda z}{F}\right)^{2} \exp \left(\frac{-\mathrm{i} \zeta \mathbf{s} \cdot \mathbf{t}}{F^{2}}\right) \exp \left(\frac{-\alpha s^{2}-\beta t^{2}}{F^{2}}\right)$,

where the following definitions have been used:

$$
\begin{gathered}
F^{2}=1+\frac{4 \alpha \beta}{\zeta^{2}}, \\
\alpha=\frac{1}{2 \sigma^{2}}, \\
\beta=\frac{1}{8 \sigma^{2}}+\frac{1}{2 \mu^{2}} .
\end{gathered}
$$

When the propagation after the DWD is considered, the expressions of the BCP matrix elements are obtained from equation (22), after substituting from equation (25) into equations (C.1)-(C.12) of appendix C, and from the latter into equation (23). The irradiance and the DoP are eventually evaluated by means of the polarization matrix, i.e., on letting $\mathbf{r}_{1}=\mathbf{r}_{2}=\mathbf{r}$ in the expression of the BCP matrix, and using the expressions given in section 2.1.

Two particular cases will be considered in the next subsections: a totally polarized (TP) and an unpolarized (UP) incident beam. In the first case, a normalized Jones vector $(\cos \theta, \sin \theta \operatorname{expi} \phi)^{\mathrm{T}}$ (where $\mathrm{T}$ stand for transposed) representing an arbitrary state of polarization specified by the angles $\theta$ and $\phi$, yields a normalized polarization matrix given by (see equations (2) and (3))

$$
\hat{P}^{\mathrm{in}}=\frac{1}{2}\left(\begin{array}{cc}
1+\cos 2 \theta & \mathrm{e}^{\mathrm{i} \phi} \sin 2 \theta \\
\mathrm{e}^{-\mathrm{i} \phi} \sin 2 \theta & 1-\cos 2 \theta
\end{array}\right),
$$

while in the second one $\hat{P}^{\text {in }}$ is proportional to the $2 \times 2$ unit matrix.

\subsection{Totally and uniformly polarized input beam}

In this case, the BCP matrix for this input beam is of the form

$$
\begin{aligned}
\hat{J}^{\mathrm{in}, \mathrm{TP}}\left(\mathbf{r}_{1}, \mathbf{r}_{2}, 0\right)= & \frac{1}{2} J_{\mathrm{sc}}\left(\mathbf{r}_{1}, \mathbf{r}_{2}, 0\right) \\
& \times\left(\begin{array}{cc}
1+\cos 2 \theta & \mathrm{e}^{\mathrm{i} \phi} \sin 2 \theta \\
\mathrm{e}^{-\mathrm{i} \phi} \sin 2 \theta & 1-\cos 2 \theta
\end{array}\right),
\end{aligned}
$$

so that, on using equation (9), the BCP across the output plane turns out to be

$\hat{J}^{\mathrm{TP}}\left(\mathbf{r}_{1}, \mathbf{r}_{2}, 0\right)=\frac{1}{2} J_{\mathrm{sc}}\left(\mathbf{r}_{1}, \mathbf{r}_{2}, 0\right)$

$$
\times\left(\begin{array}{ll}
j_{x x}^{\mathrm{TP}}\left(x_{1}, x_{2}\right) & j_{x y}^{\mathrm{TP}}\left(x_{1}, x_{2}\right) \\
j_{x y}^{\mathrm{TP}}\left(x_{2}, x_{1}\right) & j_{y y}^{\mathrm{TP}}\left(x_{1}, x_{2}\right)
\end{array}\right),
$$

with the matrix elements given by

$$
\begin{aligned}
j_{x x}^{\mathrm{TP}}\left(x_{1}, x_{2}\right)= & 2 T_{x x}^{\mathrm{D}}\left(x_{1}\right) T_{x x}^{\mathrm{D} *}\left(x_{2}\right) \cos ^{2} \theta \\
& +2 T_{x y}^{\mathrm{D}}\left(x_{1}\right) T_{x y}^{\mathrm{D} *}\left(x_{2}\right) \sin ^{2} \theta \\
& +\left[T_{x x}^{\mathrm{D}}\left(x_{1}\right) T_{x y}^{\mathrm{D} *}\left(x_{2}\right) \mathrm{e}^{\mathrm{i} \phi}\right. \\
& \left.+T_{x y}^{\mathrm{D}}\left(x_{1}\right) T_{x x}^{\mathrm{D} *}\left(x_{2}\right) \mathrm{e}^{-\mathrm{i} \phi}\right] \sin 2 \theta, \\
j_{y y}^{\mathrm{TP}}\left(x_{1}, x_{2}\right)= & 2 T_{y x}^{\mathrm{D}}\left(x_{1}\right) T_{y x}^{\mathrm{D} *}\left(x_{2}\right) \cos ^{2} \theta \\
& +2 T_{y y}^{\mathrm{D}}\left(x_{1}\right) T_{y y}^{\mathrm{D} *}\left(x_{2}\right) \sin ^{2} \theta \\
& +\left[T_{y x}^{\mathrm{D}}\left(x_{1}\right) T_{y y}^{\mathrm{D} *}\left(x_{2}\right) \mathrm{e}^{\mathrm{i} \phi}\right. \\
& \left.+T_{y y}^{\mathrm{D}}\left(x_{1}\right) T_{y x}^{\mathrm{D} *}\left(x_{2}\right) \mathrm{e}^{-\mathrm{i} \phi}\right] \sin 2 \theta, \\
j_{x y}^{\mathrm{TP}}\left(x_{1}, x_{2}\right)= & 2 T_{x x}^{\mathrm{D}}\left(x_{1}\right) T_{y x}^{\mathrm{D} *}\left(x_{2}\right) \cos ^{2} \theta \\
& +2 T_{x y}^{\mathrm{D}}\left(x_{1}\right) T_{y y}^{\mathrm{D} *}\left(x_{2}\right) \sin ^{2} \theta \\
& +\left[T_{x x}^{\mathrm{D}}\left(x_{1}\right) T_{y y}^{\mathrm{D} *}\left(x_{2}\right) \mathrm{e}^{\mathrm{i} \phi}\right. \\
& \left.+T_{x y}^{\mathrm{D}}\left(x_{1}\right) T_{y x}^{\mathrm{D} *}\left(x_{2}\right) \mathrm{e}^{-\mathrm{i} \phi}\right] \sin 2 \theta .
\end{aligned}
$$



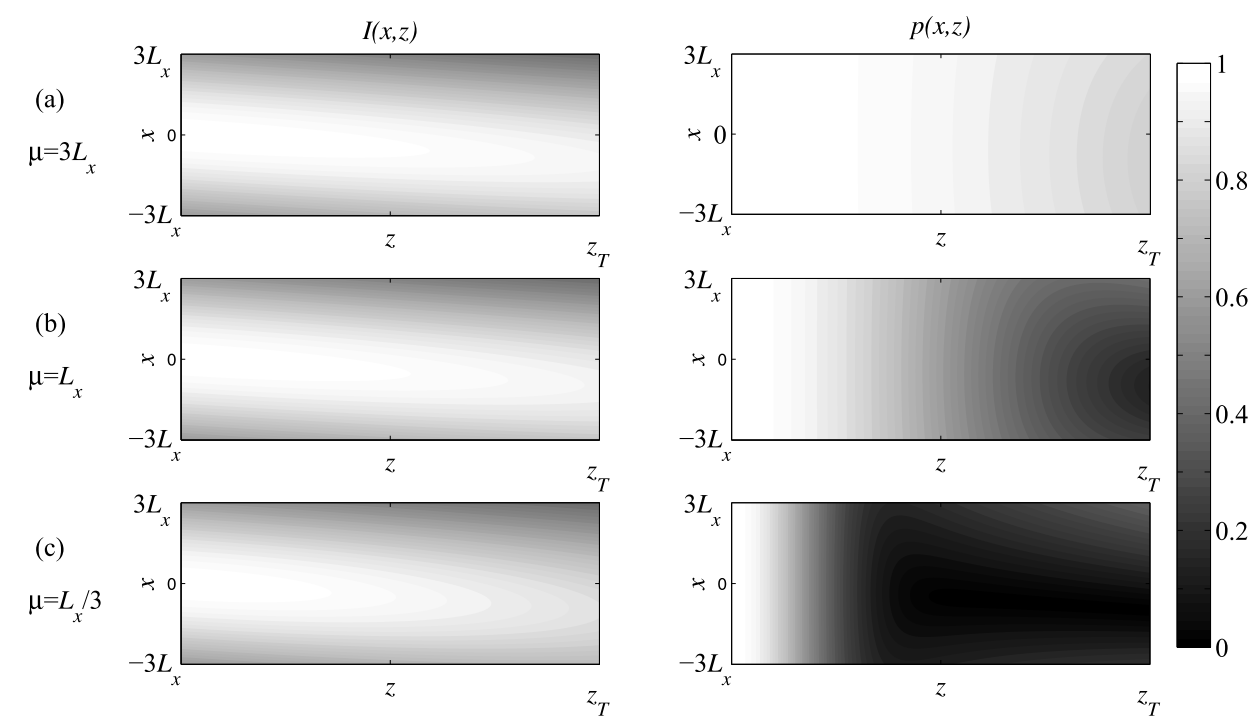

Figure 2. Normalized irradiance (left) and degree of polarization (right) for a totally linearly polarized, along $x(\theta=0)$, GSM input beam with $\sigma=3 L_{x}$ for (a) $\mu=3 L_{x}$ (upper row), (b) $\mu=L_{x}$ (middle row), and (c) $\mu=L_{x} / 3$ (lower row).

The local polarization properties of the field are obtained on letting $x_{1}=x_{2}=x$ in equations (32), (33), (34), and then using equations (31), (4) and (5). As was expected, the DoP is unity everywhere in the transverse plane of the beam, but the state of polarization varies periodically along the $x$ direction.

As far as the propagated field is concerned, its BCP matrix is calculated by using equation (22) and takes the form

$$
\begin{aligned}
\hat{J}^{\mathrm{TP}} & \left(\mathbf{r}_{1}, \mathbf{r}_{2}, z\right) \\
& =\frac{I_{0}}{2 F^{2}} \exp \left[\frac{\mathrm{i} \zeta\left(F^{2}-1\right)}{2 F^{2}}\left(r_{2}^{2}-r_{1}^{2}\right)-\frac{\alpha s_{y}^{2}+\beta t_{y}^{2}}{F^{2}}\right] \\
& \times\left(\begin{array}{cc}
h_{x x}^{\mathrm{TP}}\left(s_{x}, t_{x}, z\right) & h_{x y}^{\mathrm{TP}}\left(s_{x}, t_{x}, z\right) \\
h_{x y}^{\mathrm{TP}}\left(s_{x},-t_{x}, z\right) & h_{y y}^{\mathrm{TP}}\left(s_{x}, t_{x}, z\right)
\end{array}\right),
\end{aligned}
$$

where $s_{x}$ and $t_{x}\left(s_{y}\right.$ and $\left.t_{y}\right)$ are the $x(y)$ components of the vectors $\mathbf{s}$ and $\mathbf{t}$, and the matrix elements $h_{j k}^{\mathrm{TP}}$ are explicitly evaluated in appendix D. It can be noticed that for the DWD we are considering (i.e., oriented as in figure 1), the matrix elements $h_{j k}^{\mathrm{TP}}$ do not depend on $y$, so that the polarization properties of the field are constant along any line parallel to the $y$ axis, and the only dependence on $y$ is in the Gaussian profile. Hence, in the following, only the dependence of irradiance and DoP on $x$ and $z$ will be considered.

The optical irradiance along $x$ at any plane $z=$ const can be obtained by evaluating the trace of the polarization matrix and is

$$
I^{\mathrm{TP}}(x, z)=\frac{I_{0}}{2 F^{2}}\left[h_{x x}^{\mathrm{TP}}(x, 0, z)+h_{y y}^{\mathrm{TP}}(x, 0, z)\right],
$$

while the local degree of polarization, given by equation (4), turns out to be

$p^{\mathrm{TP}}(x, z)=\frac{\sqrt{\left[h_{x x}^{\mathrm{TP}}(x, 0, z)-h_{y y}^{\mathrm{TP}}(x, 0, z)\right]^{2}+4\left|h_{x y}^{\mathrm{TP}}(x, 0, z)\right|^{2}}}{h_{x x}^{\mathrm{TP}}(x, 0, z)+h_{y y}^{\mathrm{TP}}(x, 0, z)}$.

For the simplest cases of an incident beam linearly polarized along the $x$ axis (i.e., $\theta=0)$ or the $y$ axis $(\theta=\pi / 2)$, the optical irradiance and the DoP take the following analytical expressions:

$$
\begin{aligned}
& I_{\theta=0, \pi / 2}^{\mathrm{TP}}(x, z) \\
& \quad=\frac{I_{0}}{2 F^{2}} \exp \left(\frac{-\alpha x^{2}}{F^{2}}\right) \\
& \quad \times\left[1+\exp \left(\frac{-\alpha \gamma(\gamma \pm 2 x \zeta)}{\zeta^{2} F^{2}}\right)\right],
\end{aligned}
$$

and

$$
\begin{aligned}
p_{\theta=0, \pi / 2}^{\mathrm{TP}}(x, z) & \\
& =\frac{\sqrt{\exp \left[\frac{(\alpha-4 \beta) \gamma^{2}}{2 \zeta^{2} F^{2}}\right]+\sinh ^{2}\left[\frac{\alpha \gamma(\gamma \pm 2 x \zeta)}{2 \zeta^{2} F^{2}}\right]^{2}}}{\cosh \left[\frac{\alpha \gamma(\gamma \pm 2 x \zeta)}{\zeta^{2} F^{2}}\right]},
\end{aligned}
$$

respectively, where the $+(-)$ sign applies for linear polarization along the $x(y)$ axis. The above behaviors of the irradiance distribution and the DoP across the plane $(x, z)$ are reported in figure 2 for a beam polarized along $x$, having width $\sigma=3 L_{x}$ and several values of the coherence width: $\mu=3 L_{x}$ (a), $\mu=L_{x}$ (b), $\mu=L_{x} / 3$ (c). Here and in the following we assume a DWD made in quartz, with $\varphi=2^{\circ}$, and the wavelength is set to $0.633 \mu \mathrm{m}$. As a consequence, the periodicity of the polarization state at the exit of the DWD 


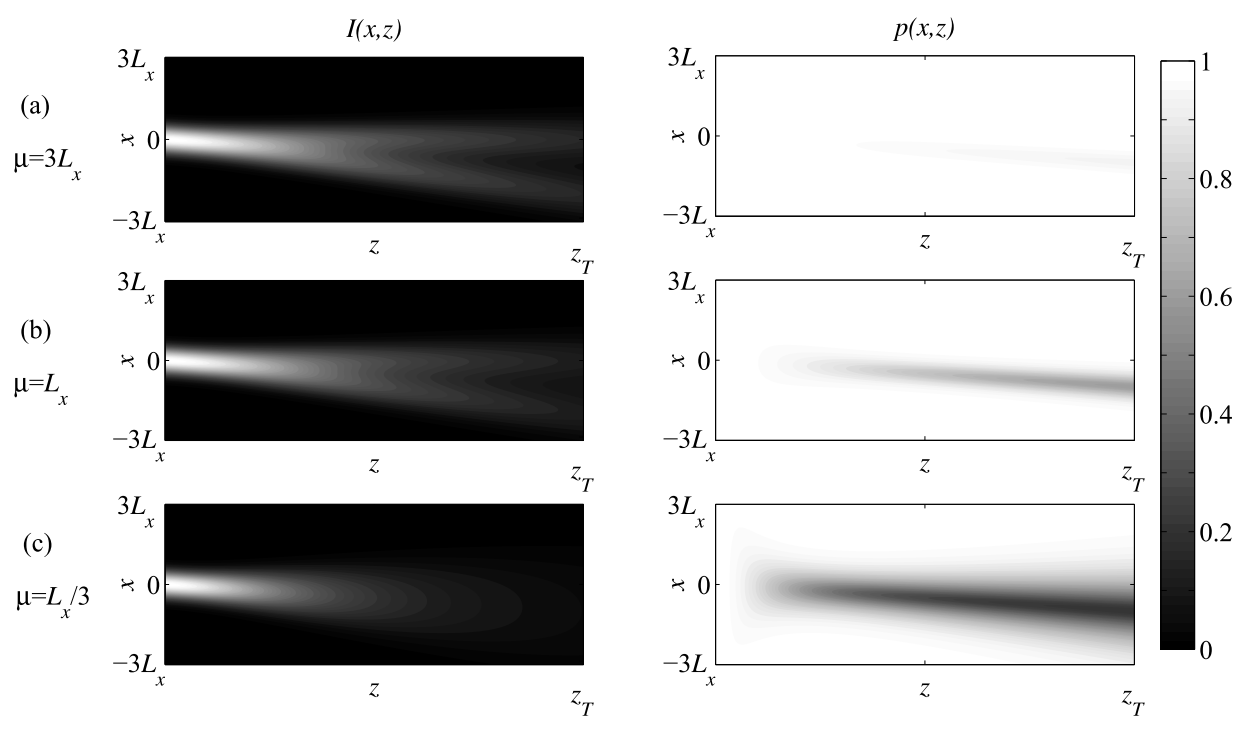

Figure 3. Normalized irradiance (left) and degree of polarization (right) for a totally linearly polarized, along $x(\theta=0$ ), GSM input beam with $\sigma=L_{x} / 3$ for (a) $\mu=3 L_{x}$ (upper row), (b) $\mu=L_{x}$ (middle row), and (c) $\mu=L_{x} / 3$ (lower row).

is $L_{x} \sim 2 \mathrm{~mm}$ (see equation (13)), corresponding to a Talbot distance $z_{\mathrm{T}} \sim 12 \mathrm{~m}$.

The plots in figure 2 show that the irradiance (left) is almost uniform beyond the DWD, while the DoP (right) is unity in a region near the DWD, but tends to vanish on increasing the propagation distance. The latter effect is more evident on decreasing the coherence width of the incoming beam.

Such behaviors can be understood if reference is made to the case of an incident plane wave having the same polarization as the beam we are considering here. The Jones vector of this plane wave is of the form $U^{\text {in }} \propto(1,0)^{\mathrm{T}}$ so that, using the Jones matrix in equation (10), the output field turns out to be of the form

$$
U^{\text {out }} \propto\left(\begin{array}{l}
1 \\
1
\end{array}\right)+\left(\begin{array}{c}
1 \\
-1
\end{array}\right) \mathrm{e}^{-\mathrm{i} \delta_{2}(x)}
$$

Since, according to our approximations, the phase $\delta_{2}$ is a linear function of $x$ (see equation (12)), the two terms in equation (40) can be recognized as being representative of two plane waves: the first one is polarized at $\pi / 4$ and propagates along the $z$ axis; the second one, with the same amplitude, has an orthogonal polarization and propagates along a tilted axis. From the values of the parameters used in these plots, the tilt angle turns out to be of the order of $2 \times 10^{-4} \mathrm{rad}$. Across any transverse plane the superposition of the two plane waves produces a polarization state that varies periodically along the $x$ direction with period $L_{x}$. This polarization pattern displaces laterally during propagation, $L_{x}$ being the lateral displacement within a Talbot distance $z_{\mathrm{T}}[9,16]$. As far as the DoP is concerned, we first note that, when the incoming field is totally polarized and perfectly coherent, the DoP at the exit of the DWD must be unity everywhere because the field is strictly deterministic. But, when the input beam is still totally polarized but partially coherent, the randomness of the field at different points may be transferred, upon propagation after the DWD, to the field components at a single point, so that the DoP may be less than unity. This is what is expected to happen when a GSM beam is used as the input beam. In particular, in figure 2(a) the coherence width is quite large, so that the DoP after the DWD is unity almost everywhere, but it decreases with $z$ when the coherence width decreases. It should be stressed that, in such a way, the DWD acts as a true depolarizer, instead of a pseudodepolarizer (see comments following equation (14)), because a perfectly polarized input beam is converted into a partially polarized output beam without the need of spatially averaging the states of polarization. In this sense, it could be considered as the spatial-coherence counterpart of a Lyot depolarizer [8, 10].

In figure 3 the same parameters as in the previous figure have been used, except for the beam width, which has been significantly reduced $\left(\sigma=L_{x} / 3\right)$. Even in this case, the incoming field is split into two beams: one of them propagates along the $z$ axis, and the other one along a different axis, corresponding to the propagation directions of the plane waves in equation (40). This effect is more evident with the highest coherence width (figure 3(a)). For lower values of $\mu$ (figures 3(b) and (c)), the two beams diverge more quickly and overlap. On the other hand, the DoP is close to unity everywhere when the input beam is almost perfectly coherent, as expected. However, when the coherence width decreases, the DoP decreases with the propagation distance, as for the previous figure, but this happens only within the superposition region of the two beams. Out of this region, when the contribution of one of the two beams is dominant, the output field is almost perfectly polarized.

More interesting behaviors can be observed when the polarization of the incident beam is linear along the bisector 

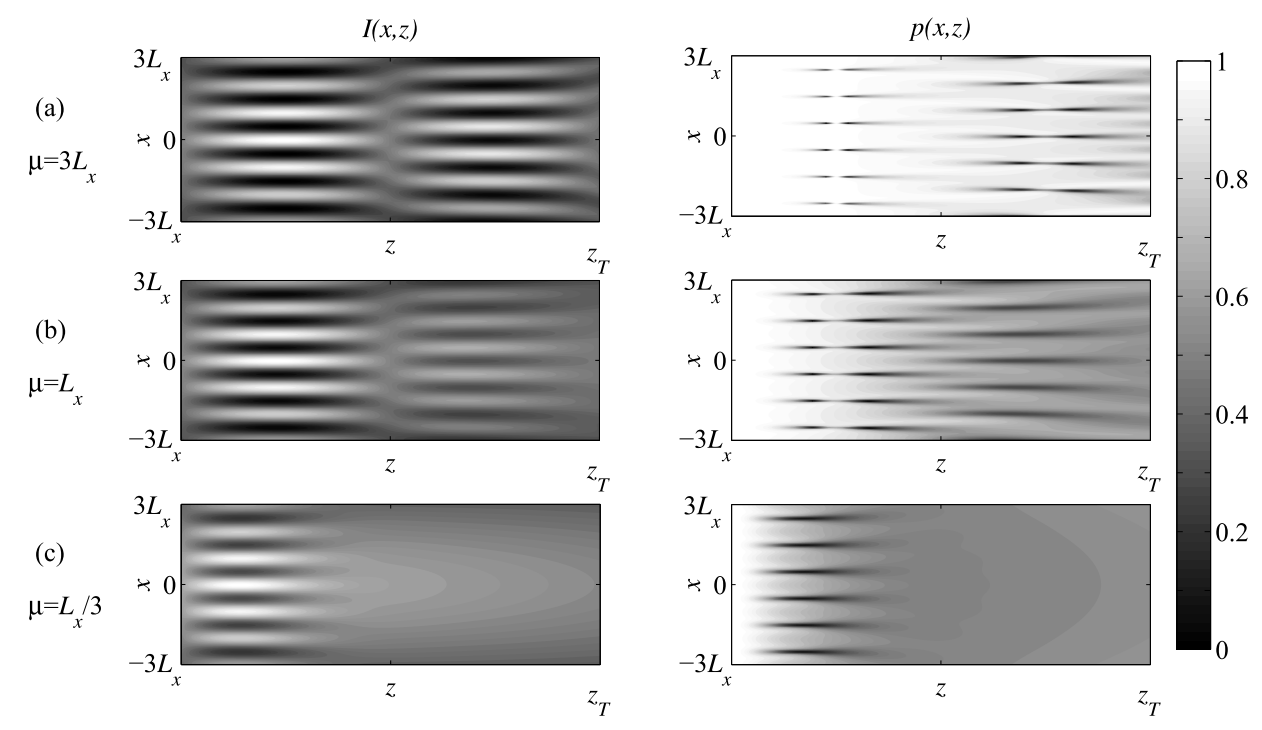

Figure 4. Normalized irradiance (left) and degree of polarization (right) for a totally linearly polarized GSM input beam with $\theta=\pi / 4$ and $\sigma=3 L_{x}$ for (a) $\mu=3 L_{x}$ (upper row), (b) $\mu=L_{x}$ (middle row), and (c) $\mu=L_{x} / 3$ (lower row).

of the $x$ and $y$ axes $(\theta=\pi / 4$ and $\phi=0)$, although in such a case the analytical expressions of the irradiance and the DoP are significantly more cumbersome. Basically the same results would be obtained for a linear polarization with $\theta=-\pi / 4$ or for a circularly polarized input beam.

The irradiance distribution and the local DoP after the DWD across the plane $(x, z)$ are shown in figures $4-6$, for a totally polarized GSM input beam with azimuth $\theta=\pi / 4$, for several combinations of profile and coherence widths. Figure 4(a) corresponds to $\sigma=\mu=3 L_{x}$. In such a case, both the beam width and the coherence width are larger than the polarization pattern period produced at the exit plane of the DWD. It can be seen that interference patterns are produced at certain transverse planes, and such patterns are almost replicated every half of the Talbot distance $\left(z_{\mathrm{T}} / 2\right)$, but with a $L_{x} / 2$ lateral displacement between two consecutive replicas $[9,16]$. The presence of such interference patterns, clearly visible at distances $z_{\mathrm{T}} / 4$ and $3 z_{\mathrm{T}} / 4$ from the DWD, can be justified in the following way.

As we did for the previous example, let us suppose that the input field is a plane wave, and that it is polarized along the bisector of the $x$ and $y$ axes. Its Jones vector is $U^{\text {in }} \propto(1,1)^{\mathrm{T}}$ so that, using equations (10)-(12), it turns out that the Jones vector at the output plane of the DWD is

$$
\begin{aligned}
U^{\text {out }} \propto & \left(\begin{array}{l}
1 \\
1
\end{array}\right)\left(1+\mathrm{e}^{-\mathrm{i} \delta_{1}(x)}\right) \\
& +\left(\begin{array}{c}
1 \\
-1
\end{array}\right)\left(1-\mathrm{e}^{-\mathrm{i} \delta_{1}(x)}\right) \mathrm{e}^{-\mathrm{i} \delta_{2}(x)} \\
= & \left(\begin{array}{l}
1 \\
1
\end{array}\right)\left(1+\mathrm{e}^{-\mathrm{i} \delta_{1}(x)}\right) \\
& +\left(\begin{array}{c}
1 \\
-1
\end{array}\right)\left(-\mathrm{e}^{-\mathrm{i}\left(\delta_{10}+\delta_{20}\right)}+\mathrm{e}^{-\mathrm{i} \delta_{2}(x)}\right) .
\end{aligned}
$$

Since, within our approximations, both the phases $\delta_{1}$ and $\delta_{2}$ are linear functions of the $x$ coordinate, this equation shows that the output field consists of four linearly polarized plane waves [29, 30]. Two of these waves have polarizations parallel to that of the input field (first term of the right hand side of equation (41)) and propagate along different directions, so that they interfere to produce a fringe pattern in the irradiance profile, with period $L_{x}$. During propagation, such a pattern moves along an axis whose orientation is determined by the propagation directions of the two waves. The other two component waves have linear polarizations as well, but with a perpendicular polarization with respect to the first two (second term of the right hand side of equation (41)). They produce an interference pattern that is transversely shifted by $L_{x} / 2$ with respect to the first pattern at the output plane and propagates along a different direction. As a consequence, no fringes at all are observed across the exit plane of the DWD when the two patterns are in phase opposition, but they appear when, after propagation through $z_{\mathrm{T}} / 4$, they superimpose in phase. Of course, the effect reproduces periodically along $z$. This simple model can explain the behavior of the irradiance profile shown in figure 4(a). As far as the DoP is concerned, the same considerations made for the previous example hold. In particular, the field is expected to be almost perfectly polarized everywhere if the input beam is almost perfectly coherent. This is what can be seen in figure 4(a): here, $\mu=3 L_{x}$ and the polarization degree is generally very close to unity, except in some regions where it takes on smaller values, and this generally happens in some transverse positions at planes around $z=z_{\mathrm{T}} / 4$.

Figure 4(b) corresponds to the same value of the beam width, $\sigma=3 L_{x}$, but the spatial coherence is reduced $\left(\mu=L_{x}\right)$. It shows that, even in this case, the irradiance interference pattern appears at $z=z_{\mathrm{T}} / 4$ and, with lower visibility, at 

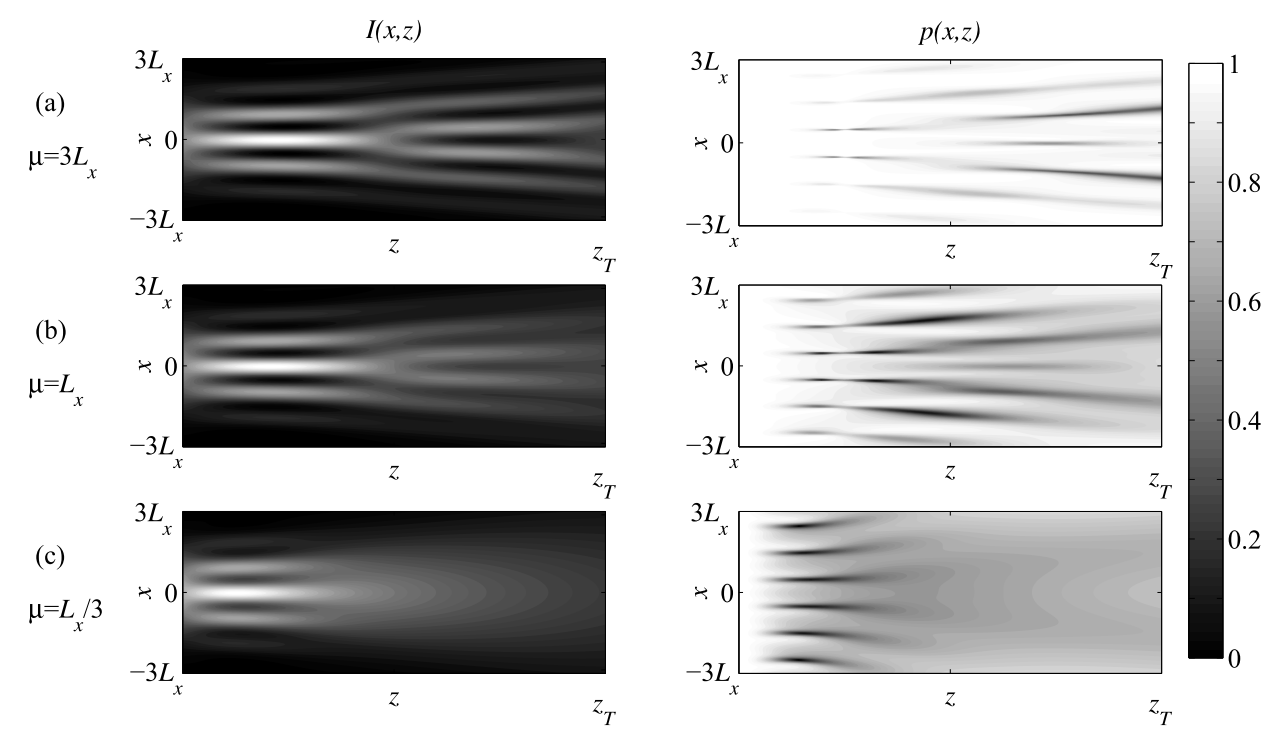

Figure 5. Normalized irradiance (left) and degree of polarization (right) for a totally linearly polarized GSM input beam with $\theta=\pi / 4$ and $\sigma=L_{x}$ for (a) $\mu=3 L_{x}$ (upper row), (b) $\mu=L_{x}$ (middle row), and (c) $\mu=L_{x} / 3$ (lower row).
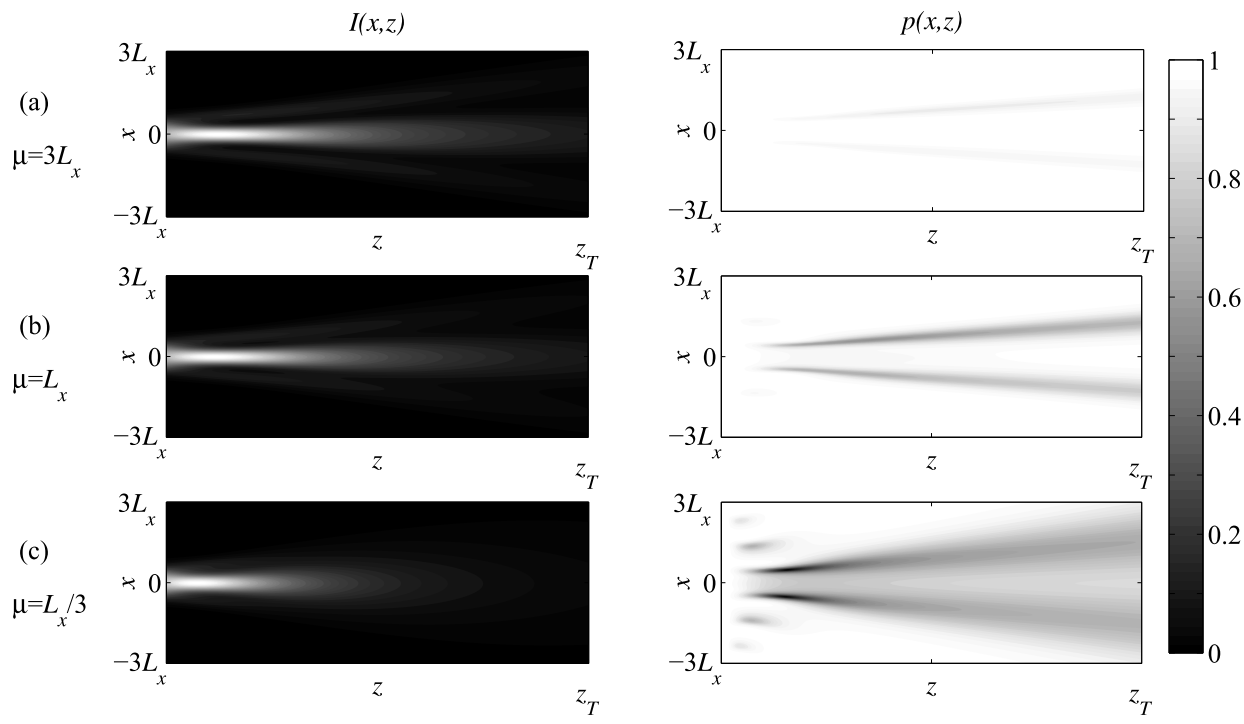

Figure 6. Normalized irradiance (left) and degree of polarization (right) for a totally linearly polarized GSM input beam with $\theta=\pi / 4$ and $\sigma=L_{x} / 3$ for (a) $\mu=3 L_{x}$ (upper row), (b) $\mu=L_{x}$ (middle row), and (c) $\mu=L_{x} / 3$ (lower row).

$3 z_{\mathrm{T}} / 4$. The degradation of the visibility, when compared to that in figure 4(a), is due to the reduction of the coherence length. The DoP is approximately unity behind the DWD but it reduces with the propagation distance. Furthermore, it presents smoother variations with respect to the previous case.

Finally, in figure $4(\mathrm{c})$, the coherence width is further reduced $\left(\mu=L_{x} / 3\right)$. The irradiance interference pattern is appreciated only around the plane $z=z_{\mathrm{T}} / 4$. For distances beyond $z_{\mathrm{T}} / 2$, the interference pattern disappears, and only the approximately transverse Gaussian profile can be appreciated. On the other hand, the DoP is nearly unity in a reduced region just behind the DWD and progressively approaches a constant value. This limiting value, reached at propagation distances well over $z_{\mathrm{T}} / 2$, depends on the characteristics of both the
DWD and the input beam. As has been previously commented, a true depolarizing effect is obtained for spatially incoherent input fields. For the present case, i.e., $\theta=\pi / 4$, the DoP for large $z$ turns out to be

$$
\begin{aligned}
& p_{\theta=\pi / 4}^{\mathrm{TP}}(x, z \rightarrow \infty)=\frac{1}{\sqrt{2}} \exp \left[\frac{(\alpha-4 \beta) \gamma^{2}}{8 \alpha \beta}\right] \\
& \quad \times\left\{\cosh \left(\frac{-\gamma^{2}}{\alpha}\right)-\cos \left(2 \delta_{10}+2 \phi\right)\right. \\
& \left.\quad+2 \exp \left[\frac{(4 \beta-\alpha) \gamma^{2}}{8 \alpha \beta}\right] \cos ^{2}\left(\delta_{10}+\phi\right)\right\}^{1 / 2} \\
& \quad \times \cosh ^{-1}\left(\frac{\gamma^{2}}{8 \beta}\right) .
\end{aligned}
$$


Similar results are shown in figure 5, but for a GSM input beam with a smaller width $\left(\sigma=L_{x}\right)$. The same general behaviors as for the previous case are observed, except for the irradiance distribution, which now presents a more evident transverse limitation.

When we consider an input beam with a transverse width smaller than the polarization periodicity $L_{x}$ (see figure 6 , with $\sigma=L_{x} / 3$ ), the interference pattern in the irradiance profile can no longer be appreciated. However, a region with higher irradiance levels is found around $z_{\mathrm{T}} / 4$. In particular, figure 6(a), which is pertinent to the case of an almost coherent incident beam, shows that three different beams are distinguishable at the output of the device. The central one, the one with the highest irradiance, propagates along the $z$ axis, while the two other beams follow the propagation directions of the plane waves in equation (41). When the incident beam is not perfectly coherent (figure 6(b), with $\mu=L_{x}$, and figure 6(c), with $\mu=L_{x} / 3$ ), each of the component beams spreads quicker and, in the overlapping region, the DoP is noticeable lower. Such an effect is better appreciated on reducing the coherence widths (figure 6(c)).

\subsection{Unpolarized input beam}

The BCP matrix of a purely unpolarized GSM input beam is of the form

$$
\hat{J}^{\mathrm{in}, \mathrm{UP}}\left(\mathbf{r}_{1}, \mathbf{r}_{2}, 0\right)=\frac{1}{2} J_{\mathrm{sc}}\left(\mathbf{r}_{1}, \mathbf{r}_{2}, 0\right)\left(\begin{array}{ll}
1 & 0 \\
0 & 1
\end{array}\right),
$$

and the corresponding BCP matrix across the output plane of the DWD turns out to be

$$
\begin{aligned}
\hat{J}^{\mathrm{UP}}\left(\mathbf{r}_{1}, \mathbf{r}_{2}, 0\right) & \\
= & \frac{1}{2} J_{\mathrm{sc}}\left(\mathbf{r}_{1}, \mathbf{r}_{2}, 0\right)\left(\begin{array}{ll}
j_{x x}^{\mathrm{UP}}\left(x_{1}, x_{2}\right) & j_{x y}^{\mathrm{UP}}\left(x_{1}, x_{2}\right) \\
j_{y x}^{\mathrm{UP}}\left(x_{2}, x_{1}\right) & j_{y y}^{\mathrm{UP}}\left(x_{1}, x_{2}\right)
\end{array}\right),
\end{aligned}
$$

where

$$
\begin{aligned}
& j_{x x}^{\mathrm{UP}}\left(x_{1}, x_{2}\right)=T_{x x}^{\mathrm{D}}\left(x_{1}\right) T_{x x}^{\mathrm{D} *}\left(x_{2}\right)+T_{x y}^{\mathrm{D}}\left(x_{1}\right) T_{x y}^{\mathrm{D} *}\left(x_{2}\right), \\
& j_{y y}^{\mathrm{UP}}\left(x_{1}, x_{2}\right)=T_{y x}^{\mathrm{D}}\left(x_{1}\right) T_{y x}^{\mathrm{D} *}\left(x_{2}\right)+T_{y y}^{\mathrm{D}}\left(x_{1}\right) T_{y y}^{\mathrm{D} *}\left(x_{2}\right), \\
& j_{x y}^{\mathrm{UP}}\left(x_{1}, x_{2}\right)=T_{x x}^{\mathrm{D}}\left(x_{1}\right) T_{y x}^{\mathrm{D} *}\left(x_{2}\right)+T_{x y}^{\mathrm{D}}\left(x_{1}\right) T_{y y}^{\mathrm{D} *}\left(x_{2}\right) .
\end{aligned}
$$

A straightforward calculation yields to a zero local degree of polarization everywhere in the transverse plane just at the exit of the DWD, as is expected for this case. Nonetheless, changes produced by the DWD in the BCP matrix elements will affect the subsequent propagation of the beam, as will be clear in the following.

For the present case, equation (22) takes the form

$$
\begin{aligned}
\hat{J}^{\mathrm{UP}} & \left(\mathbf{r}_{1}, \mathbf{r}_{2}, z\right) \\
& =\frac{I_{0}}{2 F^{2}} \exp \left[\frac{\mathrm{i} \zeta\left(F^{2}-1\right)}{2 F^{2}}\left(r_{2}^{2}-r_{1}^{2}\right)-\frac{\alpha s_{y}^{2}+\beta t_{y}^{2}}{F^{2}}\right] \\
& \times\left(\begin{array}{cc}
h_{x x}^{\mathrm{UP}}\left(s_{x}, t_{x}, z\right) & h_{x y}^{\mathrm{UP}}\left(s_{x}, t_{x}, z\right) \\
h_{x y}^{\mathrm{UP}}\left(s_{x},-t_{x}, z\right) & h_{y y}^{\mathrm{UP}}\left(s_{x}, t_{x}, z\right)
\end{array}\right),
\end{aligned}
$$

where the matrix elements $h_{j k}^{\mathrm{UP}}$ are explicitly reported in appendix E. As for the previous case, the polarization properties of the field are independent of $y$ and the irradiance presents only a Gaussian dependence on $y$, so that only the dependence of irradiance and DoP on $x$ and $z$ will be discussed in the following.

By evaluating the trace of the BCP matrix, the following optical irradiance is obtained:

$$
\begin{aligned}
I^{\mathrm{UP}}(x, z)= & \frac{I_{0}}{2 F^{2}} \exp \left(\frac{-\alpha x^{2}}{F^{2}}\right)\left[1+\exp \left(\frac{-\alpha \gamma^{2}}{\zeta^{2} F^{2}}\right)\right. \\
& \left.\times \cosh \left(\frac{2 \alpha \gamma x}{\zeta F^{2}}\right)\right],
\end{aligned}
$$

where the Gaussian dependence of the optical irradiance along the $y$ coordinate has been omitted and, by using equation (4), the DoP can be obtained as

$$
\begin{aligned}
p^{\mathrm{UP}}(x, z)= & \left\{2 \exp \left[\frac{-(\alpha+4 \beta) \gamma^{2}}{2 \zeta^{2} F^{2}}\right]\right. \\
& \times\left[\cosh \left(\frac{2 \alpha \gamma x}{\zeta F^{2}}\right)-\cos \left(\frac{\gamma^{2}}{\zeta F^{2}}\right)\right] \\
& \left.+\exp \left(\frac{-2 \alpha \gamma^{2}}{\zeta^{2} F^{2}}\right) \sinh ^{2}\left(\frac{2 \alpha \gamma x}{\zeta F^{2}}\right)\right\}^{1 / 2} \\
& \times\left[1+\exp \left(\frac{-\alpha \gamma^{2}}{\zeta^{2} F^{2}}\right)\right. \\
& \left.\times \cosh \left(\frac{2 \alpha \gamma x}{\zeta F^{2}}\right)\right]^{-1}
\end{aligned}
$$

It can be noted that the DoP is zero for $z=0$ and for $z \rightarrow \infty$, at any transverse coordinate. At the transverse coordinate $x=0$, its dependence on the $z$ coordinate takes the form

$$
\begin{aligned}
p^{\mathrm{UP}}(x=0, z)= & \exp \left[\frac{(\alpha-4 \beta) \gamma^{2}}{4 \zeta^{2} F^{2}}\right]\left|\sin \left(\frac{\gamma^{2}}{2 \zeta F^{2}}\right)\right| \\
& \times \cosh ^{-1}\left(\frac{\alpha \gamma^{2}}{2 \zeta^{2} F^{2}}\right)
\end{aligned}
$$

The behaviors of the irradiance and the DoP across the plane $(x, z)$ for a totally unpolarized GSM input beam with different coherence lengths are shown in figures 7-9 (for beam widths $\sigma=3 L_{x}, L_{x}$, and $L_{x} / 3$, respectively).

Figure 7(a) shows that, when the beam and coherence widths are large enough compared to the polarization periodicity $L_{x}$, almost the same results obtained for an incident unpolarized plane wave are reproduced $[9,16]$ : the irradiance is nearly uniform, except for the transverse Gaussian modulation, while the DoP is almost constant across any transverse plane, but varies with $z$, going from zero to nearly unity, and again to zero around $z_{\mathrm{T}} / 2$. This behavior is repeated along the $z$ axis in a quasi-periodic way. The DoP does not reach unity around $3 z_{\mathrm{T}} / 4$, as is the case for an incident plane wave, and the successive maxima reached by the DoP progressively decrease because of the finite value of the coherence area.

The periodic longitudinal modulation of the DoP can be understood on considering that a totally unpolarized incident plane wave can be thought of as the incoherent superposition of two plane waves, linearly polarized along $x$ and $y$, respectively. 

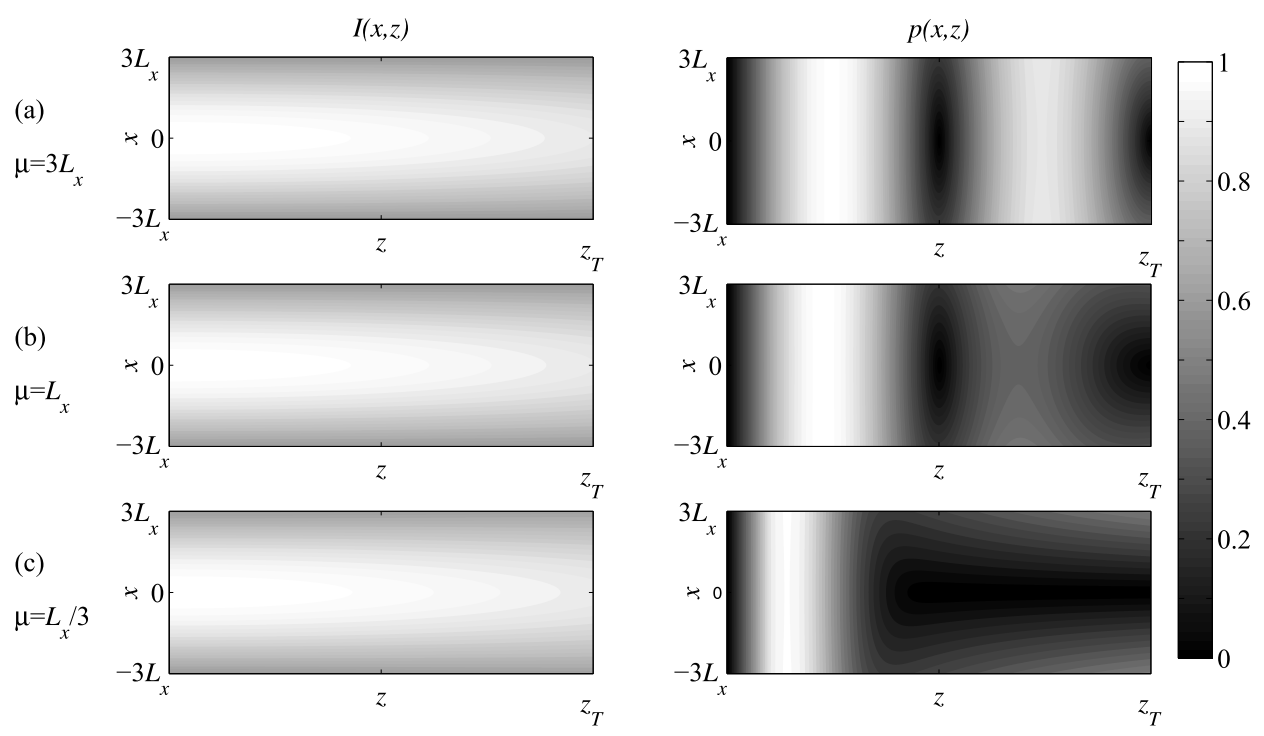

Figure 7. Normalized irradiance (left) and degree of polarization (right) for a completely unpolarized GSM input beam with $\sigma=3 L_{x}$ for (a) $\mu=3 L_{x}$ (upper row), (b) $\mu=L_{x}$ (middle row), and (c) $\mu=L_{x} / 3$ (lower row).
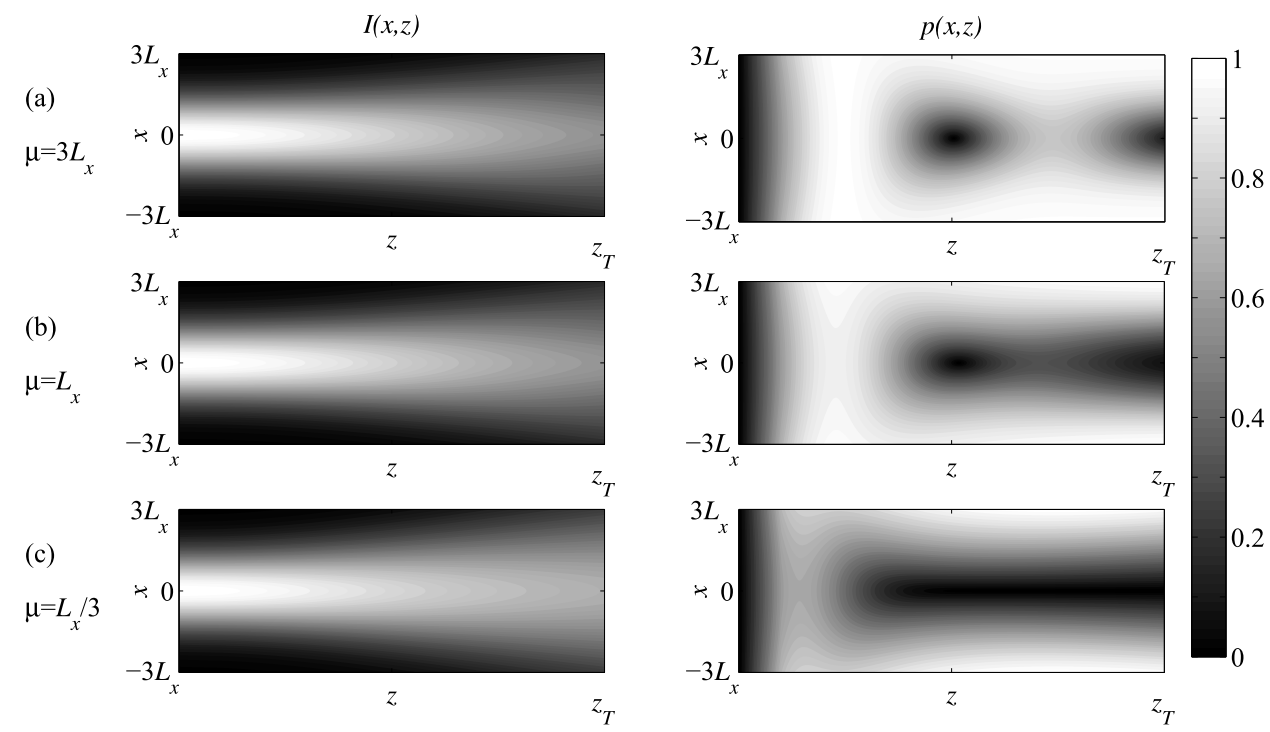

Figure 8. Normalized irradiance (left) and degree of polarization (right) for a completely unpolarized GSM input beam with $\sigma=L_{x}$ for (a) $\mu=3 L_{x}$ (upper row), (b) $\mu=L_{x}$ (middle row), and (c) $\mu=L_{x} / 3$ (lower row).

Equation (40) expresses the Jones vector of the field at the exit plane of the DWD when the input field is a plane wave linearly polarized along $x$. The corresponding polarization state varies periodically along $x$ with period $L_{x}$, and this polarization pattern shifts transversally during propagation. A similar result is obtained when the input field is a plane wave linearly polarized along $y$, but in such a case the state of polarization turns out to be orthogonal to the previous one at any $x$ position across the exit plane of the DWD and, moreover, the polarization pattern shifts in the opposite direction. The superposition of the two polarization patterns gives rise to a completely unpolarized field across the exit plane of the DWD but, due to their different shift directions, the two patterns turn out to be perfectly coincident at $z=z_{\mathrm{T}} / 4$, so that the degree of polarization reaches unity $[9,16]$. On the other hand, no irradiance interference patterns appear in this case, because the exiting beams with the same polarization state are mutually completely incoherent, while the exiting beams that are partially coherent present orthogonal polarizations. On reducing the coherence area $\left(\mu=L_{x}\right.$ in figure $\left.7(\mathrm{~b})\right)$, no remarkable effects appear on the irradiance distribution, but the behavior of the DoP changes radically, and its second maximum at $z=3 z_{\mathrm{T}} / 4$ is quite lower than at $z_{\mathrm{T}} / 4$. For $\mu=L_{x} / 3$ (figure $7(\mathrm{c})$ ), the DoP reaches a maximum, for a propagation distance slightly lower than $z_{\mathrm{T}} / 4$, and then progressively decreases to zero on increasing $z$.

In figure 8 the beam width has been reduced to $\sigma=L_{x}$, while the coherence widths are the same as for the previous figure. In this case, the spread due to diffraction is more evident, especially for lower values of the coherence width. 

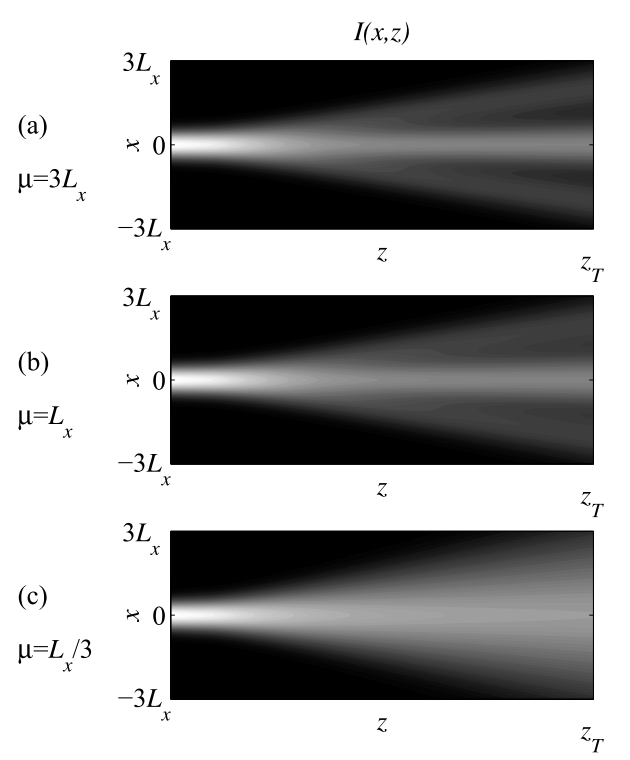
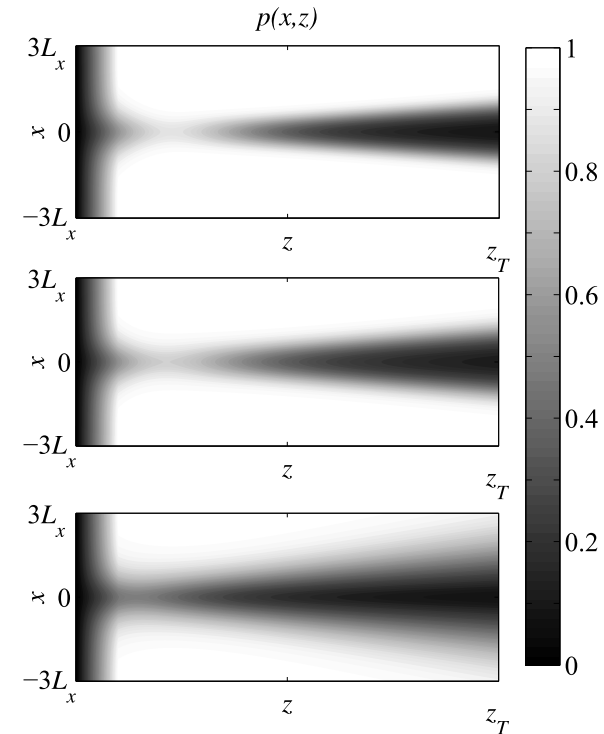

Figure 9. Normalized irradiance (left) and degree of polarization (right) for a completely unpolarized GSM input beam with $\sigma=L_{x} / 3$ for (a) $\mu=3 L_{x}$ (upper row), (b) $\mu=L_{x}$ (middle row), and (c) $\mu=L_{x} / 3$ (lower row).

The behavior of the DoP as a function of $z$ is similar to that of the previous case in the neighborhood of the $z$ axis. Out of this region, the DoP is significantly higher than zero. This can be understood, once again, on making reference to the scheme in figure 1 and equation (41). In the outer regions, in fact, the component beams do not overlap in a significant way and only one perfectly polarized beam is dominant, so that the DoP is expected to approach unity.

Finally, when the beam width is smaller than the polarization periodicity $L_{x}$ (figure 9), the irradiance splits into three parts, as was the case for a perfectly polarized input beam. This is clearly observable for $\mu=3 L_{x}$ (figure 9(a)) and $\mu=L_{x}$ (figure 9(b)), while the three component beams significantly overlap when the coherence area is the smallest $\left(\mu=L_{x} / 3\right.$, in figure 9(c)). As far as the DoP is concerned, the same considerations hold as the ones made with reference to figure 8 .

\section{Conclusions}

$\mathrm{BCP}$ formalism is used to analyze the polarization characteristics and irradiance profile of partially coherent and partially polarized beams propagating after they have passed through a non-uniform deterministic linear optical element. It has been shown that non-uniform deterministic linear optical elements transform purely polarized input fields into non-purely polarized output beams. The expression of the BCP matrix representing such field is developed for the class of optical elements described by Jones matrices whose elements are linear combinations of phases that linearly depend on the position across the optical element plane (the Jones matrices given by equation (20)). This class of optical elements include DWDs and polarization gratings.

The irradiance profile, the state of polarization and the DoP when purely polarized GSM beams impinge on a DWD and propagate after it have been analyzed in detail. Although the DoP and the irradiance just at the exit plane of the DWD are the same as those of the incoming beam, the irradiance profile and the DoP, as well as the state of polarization, vary with the propagation distance after the DWD.

When the transverse profile and the coherence area of the incident GSM beam are large enough (with respect to the transverse polarization periodicity induced by the optical element), the polarization state and the DoP of the propagated field behave almost as expected for incident plane waves presenting, in particular, the typical periodicity predicted from the Talbot effect $[9,16]$.

More complex behaviors are obtained on reducing the coherence width and/or the irradiance width of the input beam. For instance, it has been found that during propagation the DoP may tend to a constant value, lower than unity, even in the case of a totally polarized input beam. This result shows, in particular, that a DWD may act as a true depolarizer for input fields partially coherent from the spatial point of view. On the other hand, for small values of the input beam width a splitting of the beam into two or three parts (depending on the polarization state of the input beam) can be appreciated, whose superposition fixes the irradiance distribution and polarization properties of the resulting field. The propagation directions and diffraction widening of such beams depend on the DWD parameters and on the coherence width of the input field, respectively.

The present treatment can be easily extended to the case of polarization gratings $[14,15]$ and to any spatially periodic deterministic optical element.

\section{Appendix A}

Tables A.1 and A.2 collect all the symbols used throughout the text, their meaning and the first equation where they are used. 
Table A.1. Latin letters symbols and their meaning.

\begin{tabular}{lll}
\hline Symbol & Meaning & First equation \\
\hline$a_{j k}^{q}$ & Complex coefficients & $(20)$ \\
$\mathrm{D}($ superscript $)$ & Double-wedge depolarizer (DWD) characteristic & $(10)$ \\
$d_{i}$ & Mean thickness of the $i$ th wedge $(i=1,2)$ & $(11)$ \\
$E_{j}(\mathbf{r}, z ; t)$ & Electric field component along $j(j=x, y)$ & $(2)$ \\
$F^{2}$ & $1+\frac{4 \alpha \beta}{\zeta^{2}}$ & $(25)$ \\
$H_{j k}(\mathbf{s}, \mathbf{t})$ & Auxiliary function & $(18)$ \\
$h_{j k}\left(s_{x}, t_{x}, z\right)$ & Polarization part of the BCP matrix after the DWD & $(35)$ \\
$I$ & Irradiance & $(36)$ \\
$I_{0}$ & Maximum irradiance of a GSM beam & $(24)$ \\
in $($ superscript $)$ & Input beam quantity & $(6)$ \\
$\hat{J}\left(\mathbf{r}_{1}, \mathbf{r}_{2}, z\right)$ & Beam coherence-polarization (BCP) matrix & $(1)$ \\
$J_{\mathrm{sc}}\left(\mathbf{r}_{1}, \mathbf{r}_{2}, 0\right)$ & Scalar mutual intensity & $(8)$ \\
$j_{j k}^{l m}\left(\mathbf{r}_{1}, \mathbf{r}_{2}, z\right)$ & BCP elements' terms after a system defined by equation $(20)$ & $(22)$ \\
$j_{j k}\left(x_{1}, x_{2}\right)$ & Polarization part of the BCP matrix at the DWD exit plane & $(31)$ \\
$K_{z}(\mathbf{r}, \boldsymbol{\rho})$ & Direct propagator in paraxial conditions & $(15)$ \\
$L_{x}$ & Transverse period of the polarization state & $(13)$ \\
$\hat{M}^{\mathrm{D}}(x)$ & Müller matrix for a DWD & $(14)$ \\
$\hat{P}(\mathbf{r}, z)$ & Polarization matrix & $(3)$ \\
$p(\mathbf{r}, z)$ & Degree of polarization (DoP) & $(4)$ \\
$\mathbf{r}$ & Position vector in a transverse plane & $(1)$ \\
$\mathbf{S}(\mathbf{r})$ & Stokes vector & $(5)$ \\
$\hat{T}(\mathbf{r})$ & Jones matrix of a deterministic linear optical element \\
$\mathbf{s}$ & $\left(\mathbf{r}_{1}+\mathbf{r}_{2}\right) / 2$ & $(6)$ \\
$\mathbf{t}$ & $\mathbf{r}_{2}-\mathbf{r}_{1}$ & $(18)$ \\
$\mathrm{TP}($ superscript $)$ & Quantity derived for totally polarized input beams & $(18)$ \\
$U$ & Jones vector & $(29)$ \\
$\mathrm{UP}($ superscript $)$ & Quantity derived for completely unpolarized input beams & $(40)$ \\
$z_{\mathrm{T}}$ & Talbot distance & $(43)$ \\
\hline & & \\
\hline
\end{tabular}

Table A.2. Greek letter symbols and their meaning.

\begin{tabular}{lll}
\hline Symbol & Meaning & First equation \\
\hline$\alpha$ & $\frac{1}{2 \sigma^{2}}$ & $(25)$ \\
$\beta$ & $\frac{1}{8 \sigma^{2}}+\frac{1}{2 \mu^{2}}$ & $(25)$ \\
$\gamma$ & $(2 \pi|\Delta n| \tan \varphi) / \lambda$ & $(11)$ \\
$\gamma_{j k}^{q}$ & Proportionality vectors & $(20)$ \\
$\delta_{i}(x)$ & Phase difference in the $i$ th wedge $(i=1,2)$ & $(10)$ \\
$\delta_{i 0}$ & Mean phase difference in the $i$ th wedge $(i=1,2)$ & $(11)$ \\
$\Delta n$ & Birefringence & $(11)$ \\
$\zeta$ & $2 \pi /(\lambda z)$ & $(17)$ \\
$\theta, \phi$ & Angles defining the polarization state & $(29)$ \\
$\lambda$ & Wavelength & $(11)$ \\
$\mu$ & Width of the degree of coherence & $(24)$ \\
$\rho$ & Position vector across the source plane & $(15)$ \\
$\sigma^{2}$ & Variance of the irradiance profile & $(24)$ \\
$\varphi$ & Wedge angle & $(11)$ \\
\hline
\end{tabular}




\section{Appendix B}

In this appendix, the particular expressions of products $T_{j l}\left(\mathbf{r}_{1}\right) T_{k m}^{*}\left(\mathbf{r}_{2}\right)$, which are necessary to evaluate equations (7) and (9) for the case of a DWD are developed.

By using equation (10) it is obtained that

$$
\begin{aligned}
& T_{x x}^{\mathrm{D}}\left(x_{1}\right) T_{x x}^{\mathrm{D} *}\left(x_{2}\right)=\frac{1}{4}\left[1+\mathrm{e}^{-\mathrm{i} \delta_{2}\left(x_{1}\right)}\right. \\
& \left.+\mathrm{e}^{\mathrm{i} \delta_{2}\left(x_{2}\right)}+\mathrm{e}^{\mathrm{i} \delta_{2}\left(x_{2}\right)-\mathrm{i} \delta_{2}\left(x_{1}\right)}\right], \\
& T_{x y}^{\mathrm{D}}\left(x_{1}\right) T_{x x}^{\mathrm{D} *}\left(x_{2}\right)=\frac{1}{4} \mathrm{e}^{-\mathrm{i} \delta_{1}\left(x_{1}\right)}\left[1-\mathrm{e}^{-\mathrm{i} \delta_{2}\left(x_{1}\right)}\right. \\
& \left.+\mathrm{e}^{\mathrm{i} \delta_{2}\left(x_{2}\right)}-\mathrm{e}^{\mathrm{i} \delta_{2}\left(x_{2}\right)-\mathrm{i} \delta_{2}\left(x_{1}\right)}\right], \\
& T_{x x}^{\mathrm{D}}\left(x_{1}\right) T_{x y}^{\mathrm{D} *}\left(x_{2}\right)=\frac{1}{4} \mathrm{e}^{\mathrm{i} \delta_{1}\left(x_{2}\right)}\left[1+\mathrm{e}^{-\mathrm{i} \delta_{2}\left(x_{1}\right)}\right. \\
& \left.-\mathrm{e}^{\mathrm{i} \delta_{2}\left(x_{2}\right)}-\mathrm{e}^{\mathrm{i} \delta_{2}\left(x_{2}\right)-\mathrm{i} \delta_{2}\left(x_{1}\right)}\right], \\
& T_{x y}^{\mathrm{D}}\left(x_{1}\right) T_{x y}^{\mathrm{D}^{*}}\left(x_{2}\right)=\frac{1}{4} \mathrm{e}^{\mathrm{i} \delta_{1}\left(x_{2}\right)-\mathrm{i} \delta_{1}\left(x_{1}\right)}\left[1-\mathrm{e}^{-\mathrm{i} \delta_{2}\left(x_{1}\right)}\right. \\
& \left.-\mathrm{e}^{\mathrm{i} \delta_{2}\left(x_{2}\right)}+\mathrm{e}^{\mathrm{i} \delta_{2}\left(x_{2}\right)-\mathrm{i} \delta_{2}\left(x_{1}\right)}\right], \\
& T_{y x}^{\mathrm{D}}\left(x_{1}\right) T_{y x}^{\mathrm{D} *}\left(x_{2}\right)=\frac{1}{4}\left[1-\mathrm{e}^{-\mathrm{i} \delta_{2}\left(x_{1}\right)}-\mathrm{e}^{\mathrm{i} \delta_{2}\left(x_{2}\right)}\right. \\
& \left.+\mathrm{e}^{\mathrm{i} \delta_{2}\left(x_{2}\right)-\mathrm{i} \delta_{2}\left(x_{1}\right)}\right] \text {, } \\
& T_{y y}^{\mathrm{D}}\left(x_{1}\right) T_{y x}^{\mathrm{D} *}\left(x_{2}\right)=\frac{1}{4} \mathrm{e}^{-\mathrm{i} \delta_{1}\left(x_{1}\right)}\left[1+\mathrm{e}^{-\mathrm{i} \delta_{2}\left(x_{1}\right)}\right. \\
& \left.-\mathrm{e}^{\mathrm{i} \delta_{2}\left(x_{2}\right)}-\mathrm{e}^{\mathrm{i} \delta_{2}\left(x_{2}\right)-\mathrm{i} \delta_{2}\left(x_{1}\right)}\right], \\
& T_{y x}^{\mathrm{D}}\left(x_{1}\right) T_{y y}^{\mathrm{D} *}\left(x_{2}\right)=\frac{1}{4} \mathrm{e}^{\mathrm{i} \delta_{1}\left(x_{2}\right)}\left[1-\mathrm{e}^{-\mathrm{i} \delta_{2}\left(x_{1}\right)}+\mathrm{e}^{\mathrm{i} \delta_{2}\left(x_{2}\right)}\right. \\
& \left.-\mathrm{e}^{\mathrm{i} \delta_{2}\left(x_{2}\right)-\mathrm{i} \delta_{2}\left(x_{1}\right)}\right], \\
& T_{y y}^{\mathrm{D}}\left(x_{1}\right) T_{y y}^{\mathrm{D} *}\left(x_{2}\right)=\frac{1}{4} \mathrm{e}^{\mathrm{i} \delta_{1}\left(x_{2}\right)-\mathrm{i} \delta_{1}\left(x_{1}\right)}\left[1+\mathrm{e}^{-\mathrm{i} \delta_{2}\left(x_{1}\right)}\right. \\
& \left.+\mathrm{e}^{\mathrm{i} \delta_{2}\left(x_{2}\right)}+\mathrm{e}^{\mathrm{i} \delta_{2}\left(x_{2}\right)-\mathrm{i} \delta_{2}\left(x_{1}\right)}\right], \\
& T_{x x}^{\mathrm{D}}\left(x_{1}\right) T_{y x}^{\mathrm{D} *}\left(x_{2}\right)=\frac{1}{4}\left[1+\mathrm{e}^{-\mathrm{i} \delta_{2}\left(x_{1}\right)}-\mathrm{e}^{\mathrm{i} \delta_{2}\left(x_{2}\right)}\right. \\
& \left.-\mathrm{e}^{\mathrm{i} \delta_{2}\left(x_{2}\right)-\mathrm{i} \delta_{2}\left(x_{1}\right)}\right], \\
& T_{x y}^{\mathrm{D}}\left(x_{1}\right) T_{y x}^{\mathrm{D} *}\left(x_{2}\right)=\frac{1}{4} \mathrm{e}^{-\mathrm{i} \delta_{1}\left(x_{1}\right)}\left[1-\mathrm{e}^{-\mathrm{i} \delta_{2}\left(x_{1}\right)}\right. \\
& \left.-\mathrm{e}^{\mathrm{i} \delta_{2}\left(x_{2}\right)}+\mathrm{e}^{\mathrm{i} \delta_{2}\left(x_{2}\right)-\mathrm{i} \delta_{2}\left(x_{1}\right)}\right], \\
& T_{x x}^{\mathrm{D}}\left(x_{1}\right) T_{y y}^{\mathrm{D}^{*}}\left(x_{2}\right)=\frac{1}{4} \mathrm{e}^{\mathrm{i} \delta_{1}\left(x_{2}\right)}\left[1+\mathrm{e}^{-\mathrm{i} \delta_{2}\left(x_{1}\right)}\right. \\
& \left.+\mathrm{e}^{\mathrm{i} \delta_{2}\left(x_{2}\right)}+\mathrm{e}^{\mathrm{i} \delta_{2}\left(x_{2}\right)-\mathrm{i} \delta_{2}\left(x_{1}\right)}\right], \\
& T_{x y}^{\mathrm{D}}\left(x_{1}\right) T_{y y}^{\mathrm{D} *}\left(x_{2}\right)=\frac{1}{4} \mathrm{e}^{\mathrm{i} \delta_{1}\left(x_{2}\right)-\mathrm{i} \delta_{1}\left(x_{1}\right)}\left[1-\mathrm{e}^{-\mathrm{i} \delta_{2}\left(x_{1}\right)}\right. \\
& \left.+\mathrm{e}^{\mathrm{i} \delta_{2}\left(x_{2}\right)}-\mathrm{e}^{\mathrm{i} \delta_{2}\left(x_{2}\right)-\mathrm{i} \delta_{2}\left(x_{1}\right)}\right] .
\end{aligned}
$$

Due to the property $J_{y x}\left(\mathbf{r}_{1}, \mathbf{r}_{2}, 0\right)=J_{x y}^{*}\left(\mathbf{r}_{2}, \mathbf{r}_{1}, 0\right)$ [24], it is not necessary to calculate the last four terms.

\section{Appendix C}

For the case of a DWD, the terms $j_{j k}^{l m}\left(\mathbf{r}_{1}, \mathbf{r}_{2}, z\right)$ given in equation (23), which should be substituted in equation (22), can be calculated by taking into account the linearity and the displacement properties of the Fourier transform in equation (17) and using equations (B.1)-(B.12). Their expressions are

$$
\begin{aligned}
j_{x x}^{x x} & \left(\mathbf{r}_{1}, \mathbf{r}_{2}, z\right) \\
& =\frac{1}{4}\left[H_{x x}(\mathbf{s}, \mathbf{t})+\mathrm{e}^{-\mathrm{i} \delta_{20}} H_{x x}\left(\mathbf{s}+\frac{\gamma \mathbf{u}_{\mathbf{x}}}{2 \zeta}, \mathbf{t}-\frac{\gamma \mathbf{u}_{\mathbf{x}}}{\zeta}\right)\right. \\
& +\mathrm{e}^{\mathrm{i} \delta_{20}} H_{x x}\left(\mathbf{s}+\frac{\gamma \mathbf{u}_{\mathbf{x}}}{2 \zeta}, \mathbf{t}+\frac{\gamma \mathbf{u}_{\mathbf{x}}}{\zeta}\right) \\
& \left.+H_{x x}\left(\mathbf{s}+\frac{\gamma \mathbf{u}_{\mathbf{x}}}{\zeta}, \mathbf{t}\right)\right],
\end{aligned}
$$

$$
\begin{aligned}
j_{x x}^{y x} & \left(\mathbf{r}_{1}, \mathbf{r}_{2}, z\right) \\
& =\frac{1}{4}\left[\mathrm{e}^{-\mathrm{i} \delta_{10}} H_{y x}\left(\mathbf{s}-\frac{\gamma \mathbf{u}_{\mathbf{x}}}{2 \zeta}, \mathbf{t}+\frac{\gamma \mathbf{u}_{\mathbf{x}}}{\zeta}\right)\right. \\
& -\mathrm{e}^{-\mathrm{i}\left(\delta_{10}+\delta_{20}\right)} H_{y x}(\mathbf{s}, \mathbf{t}) \\
& +\mathrm{e}^{-\mathrm{i}\left(\delta_{10}-\delta_{20}\right)} H_{y x}\left(\mathbf{s}, \mathbf{t}+\frac{2 \gamma \mathbf{u}_{\mathbf{x}}}{\zeta}\right) \\
& \left.-\mathrm{e}^{-\mathrm{i} \delta_{10}} H_{y x}\left(\mathbf{s}+\frac{\gamma \mathbf{u}_{\mathbf{x}}}{2 \zeta}, \mathbf{t}+\frac{\gamma \mathbf{u}_{\mathbf{x}}}{\zeta}\right)\right],
\end{aligned}
$$$$
j_{x x}^{x y}\left(\mathbf{r}_{1}, \mathbf{r}_{2}, z\right)=\frac{1}{4}\left[\mathrm{e}^{\mathrm{i} \delta_{10}} H_{x y}\left(\mathbf{s}-\frac{\gamma \mathbf{u}_{\mathbf{x}}}{2 \zeta}, \mathbf{t}-\frac{\gamma \mathbf{u}_{\mathbf{x}}}{\zeta}\right)\right.
$$$$
+\mathrm{e}^{\mathrm{i}\left(\delta_{10}-\delta_{20}\right)} H_{x y}\left(\mathbf{s}, \mathbf{t}-\frac{2 \gamma \mathbf{u}_{\mathbf{x}}}{\zeta}\right)
$$$$
-\mathrm{e}^{\mathrm{i}\left(\delta_{10}+\delta_{20}\right)} H_{x y}(\mathbf{s}, \mathbf{t})
$$$$
\left.-\mathrm{e}^{\mathrm{i} \delta_{10}} H_{x y}\left(\mathbf{s}+\frac{\gamma \mathbf{u}_{\mathbf{x}}}{2 \zeta}, \mathbf{t}-\frac{\gamma \mathbf{u}_{\mathbf{x}}}{\zeta}\right)\right],
$$

$$
\begin{aligned}
& j_{x x}^{y y}\left(\mathbf{r}_{1}, \mathbf{r}_{2}, z\right)=\frac{1}{4}\left[H_{y y}\left(\mathbf{s}-\frac{\gamma \mathbf{u}_{\mathbf{x}}}{\zeta}, \mathbf{t}\right)\right. \\
& -\mathrm{e}^{-\mathrm{i} \delta_{20}} H_{y y}\left(\mathbf{s}-\frac{\gamma \mathbf{u}_{\mathbf{x}}}{2 \zeta}, \mathbf{t}-\frac{\gamma \mathbf{u}_{\mathbf{x}}}{\zeta}\right) \\
& \left.-\mathrm{e}^{\mathrm{i} \delta_{20}} H_{y y}\left(\mathbf{s}-\frac{\gamma \mathbf{u}_{\mathbf{x}}}{2 \zeta}, \mathbf{t}+\frac{\gamma \mathbf{u}_{\mathbf{x}}}{\zeta}\right)+H_{y y}(\mathbf{s}, \mathbf{t})\right],
\end{aligned}
$$

$j_{y y}^{x x}\left(\mathbf{r}_{1}, \mathbf{r}_{2}, z\right)$

$=\frac{1}{4}\left[H_{x x}(\mathbf{s}, \mathbf{t})-\mathrm{e}^{-\mathrm{i} \delta_{20}} H_{x x}\left(\mathbf{s}+\frac{\gamma \mathbf{u}_{\mathbf{x}}}{2 \zeta}, \mathbf{t}-\frac{\gamma \mathbf{u}_{\mathbf{x}}}{\zeta}\right)\right.$

$-\mathrm{e}^{\mathrm{i} \delta_{20}} H_{x x}\left(\mathbf{s}+\frac{\gamma \mathbf{u}_{\mathbf{x}}}{2 \zeta}, \mathbf{t}+\frac{\gamma \mathbf{u}_{\mathbf{x}}}{\zeta}\right)$

$\left.+H_{x x}\left(\mathbf{s}+\frac{\gamma \mathbf{u}_{\mathbf{x}}}{\zeta}, \mathbf{t}\right)\right]$

$j_{y y}^{y x}\left(\mathbf{r}_{1}, \mathbf{r}_{2}, z\right)$

$=\frac{1}{4}\left[\mathrm{e}^{-\mathrm{i} \delta_{10}} H_{y x}\left(\mathbf{s}-\frac{\gamma \mathbf{u}_{\mathbf{x}}}{2 \zeta}, \mathbf{t}+\frac{\gamma \mathbf{u}_{\mathbf{x}}}{\zeta}\right)\right.$

$+\mathrm{e}^{-\mathrm{i}\left(\delta_{10}+\delta_{20}\right)} H_{y x}(\mathbf{s}, \mathbf{t})$

$-\mathrm{e}^{-\mathrm{i}\left(\delta_{10}-\delta_{20}\right)} H_{y x}\left(\mathbf{s}, \mathbf{t}+\frac{2 \gamma \mathbf{u}_{\mathbf{x}}}{\zeta}\right)$

$\left.-\mathrm{e}^{-\mathrm{i} \delta_{10}} H_{y x}\left(\mathbf{s}+\frac{\gamma \mathbf{u}_{\mathbf{x}}}{2 \zeta}, \mathbf{t}+\frac{\gamma \mathbf{u}_{\mathbf{x}}}{\zeta}\right)\right]$,

$j_{y y}^{x y}\left(\mathbf{r}_{1}, \mathbf{r}_{2}, z\right)=\frac{1}{4}\left[\mathrm{e}^{\mathrm{i} \delta_{10}} H_{x y}\left(\mathbf{s}-\frac{\gamma \mathbf{u}_{\mathbf{x}}}{2 \zeta}, \mathbf{t}-\frac{\gamma \mathbf{u}_{\mathbf{x}}}{\zeta}\right)\right.$

$-\mathrm{e}^{\mathrm{i}\left(\delta_{10}-\delta_{20}\right)} H_{x y}\left(\mathbf{s}, \mathbf{t}-\frac{2 \gamma \mathbf{u}_{\mathbf{x}}}{\zeta}\right)$

$+\mathrm{e}^{\mathrm{i}\left(\delta_{10}+\delta_{20}\right)} H_{x y}(\mathbf{s}, \mathbf{t})$

$\left.-\mathrm{e}^{\mathrm{i} \delta_{10}} H_{x y}\left(\mathbf{s}+\frac{\gamma \mathbf{u}_{\mathbf{x}}}{2 \zeta}, \mathbf{t}-\frac{\gamma \mathbf{u}_{\mathbf{x}}}{\zeta}\right)\right]$,

$j_{y y}^{y y}\left(\mathbf{r}_{1}, \mathbf{r}_{2}, z\right)=\frac{1}{4}\left[H_{y y}\left(\mathbf{s}-\frac{\gamma \mathbf{u}_{\mathbf{x}}}{\zeta}, \mathbf{t}\right)\right.$

$+\mathrm{e}^{-\mathrm{i} \delta_{20}} H_{y y}\left(\mathbf{s}-\frac{\gamma \mathbf{u}_{\mathbf{x}}}{2 \zeta}, \mathbf{t}-\frac{\gamma \mathbf{u}_{\mathbf{x}}}{\zeta}\right)$

$\left.+\mathrm{e}^{\mathrm{i} \delta_{20}} H_{y y}\left(\mathbf{s}-\frac{\gamma \mathbf{u}_{\mathbf{x}}}{2 \zeta}, \mathbf{t}+\frac{\gamma \mathbf{u}_{\mathbf{x}}}{\zeta}\right)+H_{y y}(\mathbf{s}, \mathbf{t})\right]$, 


$$
\begin{aligned}
j_{x y}^{x x} & \left(\mathbf{r}_{1}, \mathbf{r}_{2}, z\right) \\
= & \frac{1}{4}\left[H_{x x}(\mathbf{s}, \mathbf{t})+\mathrm{e}^{-\mathrm{i} \delta_{20}} H_{x x}\left(\mathbf{s}+\frac{\gamma \mathbf{u}_{\mathbf{x}}}{2 \zeta}, \mathbf{t}-\frac{\gamma \mathbf{u}_{\mathbf{x}}}{\zeta}\right)\right. \\
& -\mathrm{e}^{\mathrm{i} \delta_{20}} H_{x x}\left(\mathbf{s}+\frac{\gamma \mathbf{u}_{\mathbf{x}}}{2 \zeta}, \mathbf{t}+\frac{\gamma \mathbf{u}_{\mathbf{x}}}{\zeta}\right) \\
& \left.-H_{x x}\left(\mathbf{s}+\frac{\gamma \mathbf{u}_{\mathbf{x}}}{\zeta}, \mathbf{t}\right)\right],
\end{aligned}
$$

$j_{x y}^{y x}\left(\mathbf{r}_{1}, \mathbf{r}_{2}, z\right)$

$=\frac{1}{4}\left[\mathrm{e}^{-\mathrm{i} \delta_{10}} H_{y x}\left(\mathbf{s}-\frac{\gamma \mathbf{u}_{\mathbf{x}}}{2 \zeta}, \mathbf{t}+\frac{\gamma \mathbf{u}_{\mathbf{x}}}{\zeta}\right)\right.$

$-\mathrm{e}^{-\mathrm{i}\left(\delta_{10}+\delta_{20}\right)} H_{y x}(\mathbf{s}, \mathbf{t})$

$-\mathrm{e}^{-\mathrm{i}\left(\delta_{10}-\delta_{20}\right)} H_{y x}\left(\mathbf{s}, \mathbf{t}+\frac{2 \gamma \mathbf{u}_{\mathbf{x}}}{\zeta}\right)$

$\left.+\mathrm{e}^{-\mathrm{i} \delta_{10}} H_{y x}\left(\mathbf{s}+\frac{\gamma \mathbf{u}_{\mathbf{x}}}{2 \zeta}, \mathbf{t}+\frac{\gamma \mathbf{u}_{\mathbf{x}}}{\zeta}\right)\right]$,

$j_{x y}^{x y}\left(\mathbf{r}_{1}, \mathbf{r}_{2}, z\right)$

$$
\begin{aligned}
& =\frac{1}{4}\left[\mathrm{e}^{\mathrm{i} \delta_{10}} H_{x y}\left(\mathbf{s}-\frac{\gamma \mathbf{u}_{\mathbf{x}}}{2 \zeta}, \mathbf{t}-\frac{\gamma \mathbf{u}_{\mathbf{x}}}{\zeta}\right)\right. \\
& +\mathrm{e}^{\mathrm{i}\left(\delta_{10}-\delta_{20}\right)} H_{x y}\left(\mathbf{s}, \mathbf{t}-\frac{2 \gamma \mathbf{u}_{\mathbf{x}}}{\zeta}\right) \\
& +\mathrm{e}^{\mathrm{i}\left(\delta_{10}+\delta_{20}\right)} H_{x y}(\mathbf{s}, \mathbf{t}) \\
& \left.+\mathrm{e}^{\mathrm{i} \delta_{10}} H_{x y}\left(\mathbf{s}+\frac{\gamma \mathbf{u}_{\mathbf{x}}}{2 \zeta}, \mathbf{t}-\frac{\gamma \mathbf{u}_{\mathbf{x}}}{\zeta}\right)\right],
\end{aligned}
$$

$j_{x y}^{y y}\left(\mathbf{r}_{1}, \mathbf{r}_{2}, z\right)$

$$
\begin{aligned}
& =\frac{1}{4}\left[H_{y y}\left(\mathbf{s}-\frac{\gamma \mathbf{u}_{\mathbf{x}}}{\zeta}, \mathbf{t}\right)\right. \\
& -\mathrm{e}^{-\mathrm{i} \delta_{20}} H_{y y}\left(\mathbf{s}-\frac{\gamma \mathbf{u}_{\mathbf{x}}}{2 \zeta}, \mathbf{t}-\frac{\gamma \mathbf{u}_{\mathbf{x}}}{\zeta}\right) \\
& \left.+\mathrm{e}^{\mathrm{i} \delta_{20}} H_{y y}\left(\mathbf{s}-\frac{\gamma \mathbf{u}_{\mathbf{x}}}{2 \zeta}, \mathbf{t}+\frac{\gamma \mathbf{u}_{\mathbf{x}}}{\zeta}\right)-H_{y y}(\mathbf{s}, \mathbf{t})\right] .
\end{aligned}
$$

$\left.+\mathrm{e}^{\mathrm{i} \delta_{20} H}\left(s-\frac{\gamma}{2 \zeta}, t+\frac{\gamma}{\zeta}\right)\right] \sin ^{2} \theta$

$+\frac{1}{2} H\left(s+\frac{\gamma}{\zeta}, t\right) \cos ^{2} \theta+\frac{1}{2} H\left(s-\frac{\gamma}{\zeta}, t\right) \sin ^{2} \theta$

$+\frac{1}{4}\left\{\mathrm{e}^{-\mathrm{i}\left(\delta_{10}+\phi\right)}\left[H\left(s-\frac{\gamma}{2 \zeta}, t+\frac{\gamma}{\zeta}\right)\right.\right.$

$\left.-H\left(s+\frac{\gamma}{2 \zeta}, t+\frac{\gamma}{\zeta}\right)\right]$

$+\mathrm{e}^{\mathrm{i}\left(\delta_{10}+\phi\right)}\left[H\left(s-\frac{\gamma}{2 \zeta}, t-\frac{\gamma}{\zeta}\right)\right.$

$\left.-H\left(s+\frac{\gamma}{2 \zeta}, t-\frac{\gamma}{\zeta}\right)\right]$

$+\mathrm{e}^{-\mathrm{i}\left(\delta_{10}-\delta_{20}+\phi\right)} H\left(s, t+\frac{2 \gamma}{\zeta}\right)$

$\left.+\mathrm{e}^{\mathrm{i}\left(\delta_{10}-\delta_{20}+\phi\right)} H\left(s, t-\frac{2 \gamma}{\zeta}\right)\right\} \sin 2 \theta$,

$h_{x y}^{\mathrm{TP}}(s, t, z)$

$=\frac{1}{2} H(s, t)\left[\cos 2 \theta+\mathrm{i} \sin \left(\delta_{10}+\delta_{20}+\phi\right) \sin 2 \theta\right]$

$+\frac{1}{2}\left[\mathrm{e}^{-\mathrm{i} \delta_{20}} H\left(s+\frac{\gamma}{2 \zeta}, t-\frac{\gamma}{\zeta}\right)\right.$

$\left.-\mathrm{e}^{\mathrm{i} \delta_{20} H}\left(s+\frac{\gamma}{2 \zeta}, t+\frac{\gamma}{\zeta}\right)\right] \cos ^{2} \theta$

$-\frac{1}{2}\left[\mathrm{e}^{-\mathrm{i} \delta_{20}} H\left(s-\frac{\gamma}{2 \zeta}, t-\frac{\gamma}{\zeta}\right)\right.$

$\left.-\mathrm{e}^{\mathrm{i} \delta_{20} H}\left(s-\frac{\gamma}{2 \zeta}, t+\frac{\gamma}{\zeta}\right)\right] \sin ^{2} \theta$

$-\frac{1}{2} H\left(s+\frac{\gamma}{\zeta}, t\right) \cos ^{2} \theta+\frac{1}{2} H\left(s-\frac{\gamma}{\zeta}, t\right) \sin ^{2} \theta$

$+\frac{1}{4}\left\{\mathrm{e}^{-\mathrm{i}\left(\delta_{10}+\phi\right)}\left[H\left(s-\frac{\gamma}{2 \zeta}, t+\frac{\gamma}{\zeta}\right)\right.\right.$

$\left.+H\left(s+\frac{\gamma}{2 \zeta}, t+\frac{\gamma}{\zeta}\right)\right]$

Again, it is not necessary to calculate the terms $j_{y x}^{l m}\left(\mathbf{r}_{1}, \mathbf{r}_{2}, z\right)$ because $J_{x y}\left(\mathbf{r}_{1}, \mathbf{r}_{2}, z\right)=J_{y x} *\left(\mathbf{r}_{2}, \mathbf{r}_{1}, z\right)$.

\section{Appendix D}

The matrix elements $h_{j k}^{\mathrm{TP}}(s, t, z)$ that appear in equation (35) can be calculated by substitution of equations (B.1)-(B.12) into (32)-(34) and then applying equation (22) to the particular case of a DWD. They result in

$$
\begin{aligned}
& +\mathrm{e}^{\mathrm{i}\left(\delta_{10}+\phi\right)}\left[H\left(s-\frac{\gamma}{2 \zeta}, t-\frac{\gamma}{\zeta}\right)\right. \\
& \left.+H\left(s+\frac{\gamma}{2 \zeta}, t-\frac{\gamma}{\zeta}\right)\right] \\
& -\mathrm{e}^{-\mathrm{i}\left(\delta_{10}-\delta_{20}+\phi\right)} H\left(s, t+\frac{2 \gamma}{\zeta}\right) \\
& \left.+\mathrm{e}^{\mathrm{i}\left(\delta_{10}-\delta_{20}+\phi\right)} H\left(s, t-\frac{2 \gamma}{\zeta}\right)\right\} \sin 2 \theta,
\end{aligned}
$$

$$
\begin{aligned}
& h_{x x}^{\mathrm{TP}}(s, t, z) \\
& \quad=\frac{1}{2} H(s, t)\left[1-\cos \left(\delta_{10}+\delta_{20}+\phi\right) \sin 2 \theta\right] \\
& +\frac{1}{2}\left[\mathrm{e}^{-\mathrm{i} \delta_{20}} H\left(s+\frac{\gamma}{2 \zeta}, t-\frac{\gamma}{\zeta}\right)\right. \\
& \left.\quad+\mathrm{e}^{\mathrm{i} \delta_{20}} H\left(s+\frac{\gamma}{2 \zeta}, t+\frac{\gamma}{\zeta}\right)\right] \cos ^{2} \theta \\
& \quad-\frac{1}{2}\left[\mathrm{e}^{-\mathrm{i} \delta_{20}} H\left(s-\frac{\gamma}{2 \zeta}, t-\frac{\gamma}{\zeta}\right)\right.
\end{aligned}
$$$$
h_{y y}^{\mathrm{TP}}(s, t, z)
$$$$
=\frac{1}{2} H(s, t)\left[1+\cos \left(\delta_{10}+\delta_{20}+\phi\right) \sin 2 \theta\right]
$$$$
-\frac{1}{2}\left[\mathrm{e}^{-\mathrm{i} \delta_{20} H} H\left(s+\frac{\gamma}{2 \zeta}, t-\frac{\gamma}{\zeta}\right)\right.
$$$$
\left.+\mathrm{e}^{\mathrm{i} \delta_{20} H} H\left(s+\frac{\gamma}{2 \zeta}, t+\frac{\gamma}{\zeta}\right)\right] \cos ^{2} \theta
$$$$
+\frac{1}{2}\left[\mathrm{e}^{-\mathrm{i} \delta_{20}} H\left(s-\frac{\gamma}{2 \zeta}, t-\frac{\gamma}{\zeta}\right)\right.
$$ 


$$
\begin{aligned}
& \left.+\mathrm{e}^{\mathrm{i} \delta_{20} H}\left(s-\frac{\gamma}{2 \zeta}, t+\frac{\gamma}{\zeta}\right)\right] \sin ^{2} \theta \\
& +\frac{1}{2} H\left(s+\frac{\gamma}{\zeta}, t\right) \cos ^{2} \theta+\frac{1}{2} H\left(s-\frac{\gamma}{\zeta}, t\right) \sin ^{2} \theta \\
& +\frac{1}{4}\left\{\mathrm { e } ^ { - \mathrm { i } ( \delta _ { 1 0 } + \phi ) } \left[H\left(s-\frac{\gamma}{2 \zeta}, t+\frac{\gamma}{\zeta}\right)\right.\right. \\
& \left.-H\left(s+\frac{\gamma}{2 \zeta}, t+\frac{\gamma}{\zeta}\right)\right] \\
& +\mathrm{e}^{\mathrm{i}\left(\delta_{10}+\phi\right)}\left[H\left(s-\frac{\gamma}{2 \zeta}, t-\frac{\gamma}{\zeta}\right)\right. \\
& \left.-H\left(s+\frac{\gamma}{2 \zeta}, t-\frac{\gamma}{\zeta}\right)\right] \\
& -\mathrm{e}^{-\mathrm{i}\left(\delta_{10}-\delta_{20}+\phi\right)} H\left(s, t+\frac{2 \gamma}{\zeta}\right) \\
& \left.-\mathrm{e}^{\mathrm{i}\left(\delta_{10}-\delta_{20}+\phi\right)} H\left(s, t-\frac{2 \gamma}{\zeta}\right)\right\} \sin 2 \theta .
\end{aligned}
$$

In these expressions $H(s, t)$ is given by equation (25) for the particular case of a GSM input beam.

\section{Appendix E}

The matrix elements $h_{j k}^{\mathrm{UP}}(s, t, z)$ that appear in equation (44) can be calculated by substitution of equations (B.1)-(B.12) into equations (45)-(47) and then applying equation (22) to the particular case of a DWD. They can be written as

$$
\begin{aligned}
& h_{x x}^{\mathrm{UP}}(s, t, z) \\
& =\frac{1}{2} H(s, t)+\frac{1}{4} H\left(s+\frac{\gamma}{\zeta}, t\right)+\frac{1}{4} H\left(s-\frac{\gamma}{\zeta}, t\right) \\
& +\frac{1}{4}\left[\mathrm{e}^{-\mathrm{i} \delta_{20} H}\left(s+\frac{\gamma}{2 \zeta}, t-\frac{\gamma}{\zeta}\right)\right. \\
& \left.+\mathrm{e}^{\mathrm{i} \delta_{20} H} H\left(s+\frac{\gamma}{2 \zeta}, t+\frac{\gamma}{\zeta}\right)\right] \\
& -\frac{1}{4}\left[\mathrm{e}^{-\mathrm{i} \delta_{20}} H\left(s-\frac{\gamma}{2 \zeta}, t-\frac{\gamma}{\zeta}\right)\right. \\
& \left.+\mathrm{e}^{\mathrm{i} \delta_{20} H} H\left(s-\frac{\gamma}{2 \zeta}, t+\frac{\gamma}{\zeta}\right)\right], \\
& h_{x y}^{\mathrm{UP}}(s, t, z) \\
& =\frac{1}{4}\left[\mathrm{e}^{-\mathrm{i} \delta_{20}} H\left(s+\frac{\gamma}{2 \zeta}, t-\frac{\gamma}{\zeta}\right)\right. \\
& \left.-\mathrm{e}^{\mathrm{i} \delta_{20} H} H\left(s+\frac{\gamma}{2 \zeta}, t+\frac{\gamma}{\zeta}\right)\right] \\
& -\frac{1}{4}\left[\mathrm{e}^{-\mathrm{i} \delta_{20}} H\left(s-\frac{\gamma}{2 \zeta}, t-\frac{\gamma}{\zeta}\right)\right. \\
& \left.-\mathrm{e}^{\mathrm{i} \delta 20} H\left(s-\frac{\gamma}{2 \zeta}, t+\frac{\gamma}{\zeta}\right)\right] \\
& -\frac{1}{4} H\left(s+\frac{\gamma}{\zeta}, t\right)+\frac{1}{4} H\left(s-\frac{\gamma}{\zeta}, t\right),
\end{aligned}
$$

$$
\begin{aligned}
h_{y y}^{\mathrm{UP}} & (s, t, z) \\
& =\frac{1}{2} H(s, t)+\frac{1}{4} H\left(s+\frac{\gamma}{\zeta}, t\right)+\frac{1}{4} H\left(s-\frac{\gamma}{\zeta}, t\right) \\
& -\frac{1}{4}\left[\mathrm{e}^{-\mathrm{i} \delta_{20}} H\left(s+\frac{\gamma}{2 \zeta}, t-\frac{\gamma}{\zeta}\right)\right. \\
& \left.+\mathrm{e}^{\mathrm{i} \delta_{20}} H\left(s+\frac{\gamma}{2 \zeta}, t+\frac{\gamma}{\zeta}\right)\right] \\
& +\frac{1}{4}\left[\mathrm{e}^{-\mathrm{i} \delta_{20}} H\left(s-\frac{\gamma}{2 \zeta}, t-\frac{\gamma}{\zeta}\right)\right. \\
& \left.+\mathrm{e}^{\mathrm{i} \delta_{20}} H\left(s-\frac{\gamma}{2 \zeta}, t+\frac{\gamma}{\zeta}\right)\right] .
\end{aligned}
$$

In these expressions $H(s, t)$ is given by equation (25) for the particular case of a GSM input beam.

\section{References}

[1] McGuire J P and Chipman R A 1990 Analysis of spatial pseudodepolarizers in imaging systems Opt. Eng. 29 1478-84

[2] El Sherif M, Khalil M S, Khodeir S and Nagib N 1996 Simple depolarizers for spectrophotometric measurements of anisotropic samples Opt. Laser Technol. 28 561-3

[3] Biener G, Niv A, Kleiner V and Hasman E 2003 Computer-generated infrared depolarizer using space-variant subwavelength dielectric gratings Opt. Lett. 28 1400-2

[4] Honma M and Nose T 2004 Liquid-crystal depolarizer consisting of randomly aligned hybrid orientation domains Appl. Opt. 43 4667-71

[5] Vartiainen I, Tervo J and Kuittinen M 2009 Depolarization of quasi-monochromatic light by thin resonant gratings $O p t$. Lett. 34 1648-50

[6] Bagan V A, Davydov B L and Samartsev I E 2009 Characteristics of Cornu depolarisers made from quartz and paratellurite optically active crystals Quantum Electron. 39 73-8

[7] Vena C, Versace C, Strangi G and Bartolino R 2009 Light depolarization by non-uniform polarization distribution over a beam cross section J. Opt. A: Pure Appl. Opt. 11125704

[8] de Sande J C G, Piquero G and Teijeiro C 2012 Polarization changes at Lyot depolarizer output for different types of input beams J. Opt. Soc. Am. A 29 278-84

[9] de Sande J C G, Santarsiero M, Piquero G and Gori F 2012 Longitudinal polarization periodicity of unpolarized light passing through a double wedge depolarizer Opt. Express 20 27348-60

[10] Makowski P L, Szymanski M Z and Domanski A W 2012 Lyot depolarizer in terms of the theory of coherence-description for light of any spectrum Appl. Opt. 51 626-34

[11] Santarsiero M 2007 Polarization invariance in a Young interferometer J. Opt. Soc. Am. A 24 3493-9

[12] Gori F, Tervo J and Turunen J 2009 Correlation matrices of completely unpolarized beams Opt. Lett. 34 1447-9

[13] Hassinen T, Tervo J and Friberg A T 2013 Purity of partial polarization in the frequency and time domains Opt. Lett. 38 1221-3

[14] Piquero G, Borghi R and Santarsiero M 2001 Gaussian Schell-model beams propagating through polarization gratings J. Opt. Soc. Am. A 18 1399-405 
[15] Piquero G, Borghi R, Mondello A and Santarsiero M 2001 Far field of beams generated by quasi-homogeneous sources passing through polarization gratings Opt. Commun. $195339-50$

[16] Santarsiero M, de Sande J C G, Piquero G and Gori F 2013 Coherence-polarization properties of fields radiated from transversely periodic electromagnetic sources $J$. Opt. 15055701

[17] Mandel L and Wolf E 1995 Optical Coherence and Quantum Optics (Cambridge: Cambridge University Press)

[18] Gori F, Santarsiero M, Piquero G, Borghi R, Mondello A and Simon R 2001 Partially polarized Gaussian Schell-model beams J. Opt. A: Pure Appl. Opt. 3 1-9

[19] Piquero G, Gori F, Romanini P, Santarsiero M, Borghi R and Mondello A 2002 Synthesis of partially polarized Gaussian Schell-model sources Opt. Commun. 208 9-16

[20] Shirai T, Korotkova O and Wolf E 2005 A method of generating electromagnetic Gaussian Schell-model beams J. Opt. A: Pure Appl. Opt. 7 232-7

[21] Wolf E 2007 Introduction to the Theory of Coherence and Polarization of Light (Cambridge: Cambridge University Press)

[22] Martínez-Herrero R, Mejías P M and Piquero G 2009 Characterization of Partially Polarized Light Fields (Springer Series in Optical Sciences vol 147) (Berlin: Springer)

[23] Gori F 1998 Matrix treatment for partially polarized, partially coherent beams Opt. Lett. 23 241-3

[24] Gori F, Santarsiero M, Vicalvi S, Borghi R and Guattari G 1998 Beam coherence polarization matrix J. Eur. Opt. Soc. A: Pure Appl. Opt. 7 941-51
[25] Gori F, Santarsiero M, Borghi R and Guattari G 1999 Irradiance of partially polarized beams in a scalar treatment Opt. Commun. 163 159-63

[26] Wolf E 2003 Unified theory of coherence and polarization of random electromagnetic beams Phys. Lett. A 312 263-7

[27] Born M and Wolf E 1999 Principles of Optics (Cambridge: Cambridge University Press) 7th expanded

[28] Simon R 1990 Nondepolarizing systems and degree of polarization Opt. Commun. 77 349-54

[29] Alekseeva L V, Povkh I V, Stroganov V I, Kidyarov B I and Pasko P G 2002 Four-ray splitting in optical crystals J. Opt Technol. 39 441-3

[30] Kuznetsov V, Faleiev D, Savin E and Lebedev V 2009 Crystal-based device for combining light beams Opt. Lett. 34 2856-7

[31] Arrizón V, Tepichin E, Ortiz-Gutiérrez M and Lohmann A W 1996 Fresnel diffraction at $1 / 4$ of the Talbot distance of an anisotropic grating Opt. Commun. 127 171-5

[32] Fujiwara H 2007 Spectroscopic Ellipsometry: Principles and Applications. Appendix A (Chichester: Wiley) pp 353-5

[33] Simon B N, Simon S, Gori F, Santarsiero M, Borghi R, Mukunda N and Simon R 2010 Nonquantum entanglement resolves a basic issue in polarization optics Phys. Rev. Lett. 104023901

[34] Chipman R A 2005 Depolarization index and the average degree of polarization Appl. Opt. 44 2490-5

[35] Gori F 1983 Mode propagation of the field generated by Collett-Wolf Schell-model sources Opt. Commun. 46 149-54 